\title{
Spin and lattice dynamics in the two-singlet system $\mathrm{Tb}_{3} \mathrm{Ga}_{5} \mathrm{O}_{12}$
}

\author{
S. Petit ${ }^{*}$ and F. Damay $\odot$ \\ Laboratoire Léon Brillouin, Université Paris-Saclay, CNRS, CEA, CEA-Saclay, F-91191 Gif-sur-Yvette, France \\ Q. Berrod $\odot$ \\ IRIG-SyMMES, Université Grenoble Alpes, CNRS, CEA, 38000 Grenoble, France \\ and Institut Laue-Langevin, 6 rue Jules Horowitz, BP 156, 38042 Grenoble CEDEX 9, France \\ J. M. Zanotti \\ Laboratoire Léon Brillouin, Université Paris-Saclay, CNRS, CEA, CEA-Saclay, F-91191 Gif-sur-Yvette, France \\ and Institut Laue-Langevin, 6 rue Jules Horowitz, BP 156, 38042 Grenoble CEDEX 9, France
}

(Received 21 October 2020; revised 14 December 2020; accepted 18 December 2020; published 12 January 2021)

\begin{abstract}
We address the issue of the origin of the phonon thermal Hall effect in $\mathrm{Tb}_{3} \mathrm{Ga}_{5} \mathrm{O}_{12}$, an intriguing property presumed to originate from magnetoelastic properties, and magnified in this compound by the non-Kramers nature of $\mathrm{Tb}^{3+}$ ions. Using neutron scattering, we have explored both the spin and lattice dynamics of $\mathrm{Tb}_{3} \mathrm{Ga}_{5} \mathrm{O}_{12}$. Our experimental results show that the transition toward the magnetic ground state, below $T_{N}=280 \mathrm{mK}$, is driven by the softening of an exciton, as expected in a two-singlet system like $\mathrm{Tb}_{3} \mathrm{Ga}_{5} \mathrm{O}_{12}$. Low-energy excitations in the ordered phase are still excitons, whose dispersion throughout the Brillouin zone is driven by magnetic interactions. We have also discovered a mixing between specific phonon and exciton modes, this hybridization being evidenced through an intensity anomaly of the transverse acoustic phonons, as they cross low-energy crystal field excitations. Those experimental results can be comprehended by random phase approximation calculations, involving a Hamiltonian based on crystal electric field, dipolar interactions, and a coupling between phonons and the quadrupolar $4 f$ electronic density.
\end{abstract}

DOI: 10.1103/PhysRevResearch.3.013030

\section{INTRODUCTION}

The phonon thermal Hall effect (PTHE), an enigmatic thermal analog of the electronic Hall effect, is characterized by a heat flow in a direction perpendicular to both an applied magnetic field and a thermal gradient. It was actually discovered more than a decade ago, in the paramagnetic insulator garnet $\mathrm{Tb}_{3} \mathrm{Ga}_{5} \mathrm{O}_{12}$ [1,2]. In this very case, it was proposed to rely on a large magnetoelastic coupling, that is, on the interaction between an elastic strain and the electronic distribution of the $4 f$ orbital moments [3]. However, the scope of this result was very quickly recognized and extended to other cases, leading to an extensive exploration of the heat-carrying properties of nontrivial excitations in a variety of other systems, such as insulating quantum magnets [4], frustrated magnetic insulators [5], spin-liquid candidates [6-8], or multiferroics [9].

Among the several possible origins for the PTHE in $\mathrm{Tb}_{3} \mathrm{Ga}_{5} \mathrm{O}_{12}$ (TbGG) that have been proposed and discussed, one has invoked a Raman-type interaction between phonons and large spins (spin-phonon coupling) [10-12], a Berry curvature of phonon bands $[13,14]$, or resonant skew scatter-

\footnotetext{
*sylvain.petit@cea.fr

Published by the American Physical Society under the terms of the Creative Commons Attribution 4.0 International license. Further distribution of this work must maintain attribution to the author(s) and the published article's title, journal citation, and DOI.
}

ing of phonons [15]. All those mechanisms involve a large magnetoelastic coupling [16,17], a property which has been repeatedly inferred from a variety of experiments in TbGG, through the softening at low temperature of its longitudinal and transverse elastic constants [16], an acoustic Faraday effect $[16,18,19]$, or a strong scattering of acoustic phonons by $\mathrm{Tb}^{3+}$ ions [20,21].

Surprisingly, although magnetoelastic coupling is arguably a cornerstone for a proper understanding of the PTHE in TbGG, there has not been any direct microscopic evidence for it yet. Furthermore, TbGG is well known to be a two-singlet system [22], yet, despite this nonmagnetic ground state, it orders magnetically at $T_{N} \approx 0.28 \mathrm{~K}[23,24]$. The magnetic order is thus expected to be of the "induced moment" type $[23,25,26]$, a feature which remains to be seen experimentally in TbGG.

This motivates the present neutron scattering study, whose aim is a comprehensive description of both the spin and lattice dynamics in this compound. Our experimental results show that the transition toward the magnetic ground state is driven by the softening of an exciton, as expected in a two-singlet system like TbGG. The spin dynamics can be reasonably well modeled by random phase approximation (RPA) calculations, involving a Hamiltonian based on crystal electric field and dipolar interactions. Furthermore, focusing on lattice dynamics, we have discovered that TbGG hosts hybrid phonon and exciton modes, this hybridization being evidenced through an intensity anomaly of the transverse acoustic phonons, as they cross low-energy crystal field excitations. We contend this 


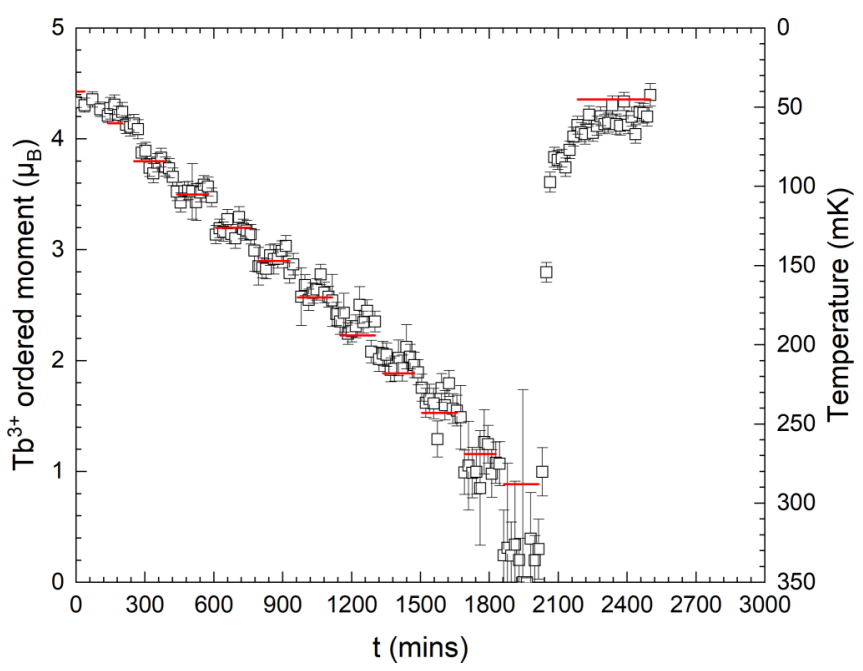

FIG. 1. Evolution of the ordered magnetic moment of $\mathrm{Tb}^{3+}$ in polycrystalline $\mathrm{Tb}_{3} \mathrm{Ga}_{5} \mathrm{O}_{12}$ versus time (left axis; from Rietveld refinements of low-statistic data). The corresponding temperature sequence (versus time) is pictured by red horizontal bars (right axis). Each horizontal bar corresponds to a time period in which the temperature is constant (i.e., outside a ramp).

anomaly to be the microscopic signature of a magnetoelastic coupling. This conclusion is further supported by including a phonon-quadrupole coupling term in the RPA calculations. These findings should prove elemental in the understanding of the macroscopic properties of $\mathrm{Tb}_{3} \mathrm{Ga}_{5} \mathrm{O}_{12}$ and more generally of the PTHE.

\section{EXPERIMENTAL METHODS}

\section{A. Sample preparation}

The $\mathrm{Tb}_{3} \mathrm{Ga}_{5} \mathrm{O}_{12}$ (TbGG) powder was synthesized from a mixture of stoichiometric amounts of $\mathrm{Tb}_{2} \mathrm{O}_{3}$ and $\mathrm{Ga}_{2} \mathrm{O}_{3}$, previously dried at $500{ }^{\circ} \mathrm{C}$, pressed into pellets and heated up to $1200^{\circ} \mathrm{C}$ for $48 \mathrm{~h}$, with regular regrindings. Formation of the pure phases was confirmed by laboratory $\mathrm{x}$-ray powder diffraction. The sample crystallizes in the expected cubic space group $I a \overline{3} d$, with $a \approx 12.35 \AA$ in agreement with literature results [27]. The single TbGG crystal $(\varnothing=10 \times 20 \mathrm{~mm})$ was bought from Northrop Grumman, USA.

\section{B. Neutron powder diffraction}

Neutron powder diffraction (NPD) versus temperature was performed on the G4.1 diffractometer $(\lambda=2.426 \AA)$ at LLB-Orphée (France). Diffractograms were recorded between $45 \mathrm{mK}$ and $290 \mathrm{mK}$, in a dilution fridge environment. To ensure proper thermalization of the sample at subkelvin temperatures, the sample was set in a dedicated vanadium cell filled with 4 bars ${ }^{4} \mathrm{He}$ gas and left for 6 hours at $45 \mathrm{mK}$ before the start of the experiment. The temperature sequence is illustrated in Fig. 1 (right axis), and corresponds to a dozen temperature steps, increasing $T$ from $45 \mathrm{mK}$ to $288 \mathrm{mK}$, before cooling down to base temperature $(45 \mathrm{mK}$ ) again (with the neutron beam always on). Between six and eight 15-min diffractograms were recorded during each temperature plateau
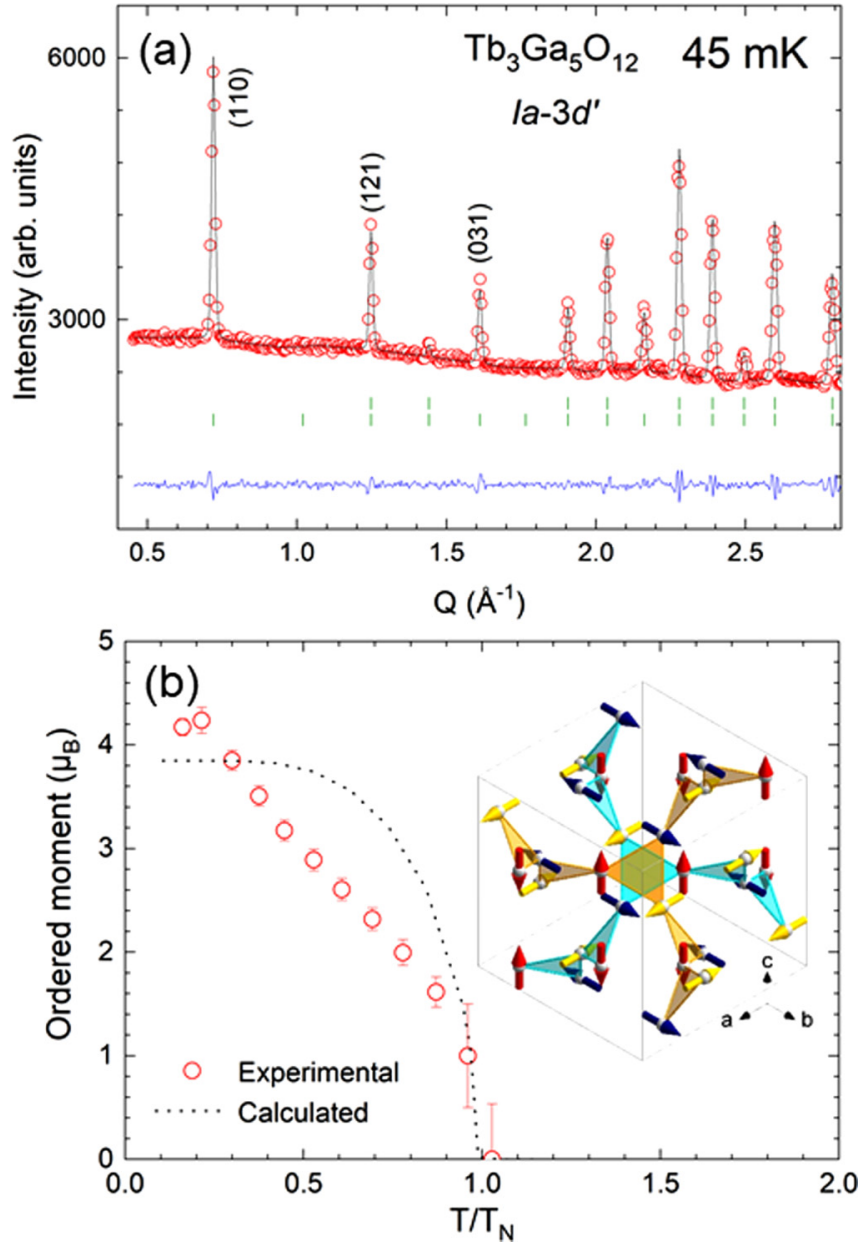

FIG. 2. (a) Rietveld refinement profile of the TbGG neutron diffraction pattern at $45 \mathrm{mK}$, using the magnetic space group $I a \overline{3} d$. (Experimental: empty red circles, calculated: black line, Bragg positions (crystal + magnetic contributions): green ticks. The difference between the experimental and calculated profiles is displayed at the bottom of the graph as a blue continuous line. (b) Reduced temperature evolution of the $\mathrm{Tb}^{3+}$ ordered magnetic moment, obtained from Rietveld refinements of the neutron diffraction data (open red symbols). The dotted line shows the corresponding calculation, based on the singlet-singlet model described in the main text. Inset of (b): Multiaxis AFA magnetic order, with the three magnetic sublattices having moments parallel or antiparallel to the cubic axis $(a, b, c$ in yellow, blue, and red, respectively).

to provide high-statistic data [such as shown in Fig. 2(a)] for subsequent Rietveld analysis. As illustrated in Fig. 1, the ordered magnetic moment extracted from the 15-min NPD data follows the temperature sequence perfectly, thus confirming the proper thermalization of the sample.

Rietveld refinements were performed with the FullProf program [28]. Symmetry analysis was carried out using the FullProf Suite software and the Bilbao Crystallographic Server $[29,30]$. No sign of partial substitution of $\mathrm{Tb}$ on the $\mathrm{Ga}$ octahedral site (and vice versa) or deviation from the nominal oxygen stoichiometry was evidenced within the resolution of the experiment $(\leqslant 3 \%)$. 


\section{Inelastic neutron scattering}

Inelastic neutron scattering (INS) measurements were performed on the thermal triple-axis 2T spectrometer (LLBOrphée, France). Standard conditions were used, with PG002 as monochromator and analyzer, using a fixed constant wave vector $k_{f}=2.662 \AA^{-1}$, along with an 8-cm-thick PG filter on the scattered beam to eliminate harmonics. Experiments were carried out with open collimations, in combination with a vertically and horizontally bent monochromator and analyzer, to optimize the flux at the sample position. This setup yields an energy resolution $\Delta E \approx 1.2 \mathrm{meV}$. The TbGG powder sample was put inside an aluminum holder. The single-crystal sample was wrapped in an aluminum foil, aligned with the [110] direction vertical, and attached to the cold finger of a closed-cycle cryostat.

Time-of-flight (TOF) INS experiments were also performed on the TbGG single crystal, using the cold spectrometer IN6-SHARP (ILL, France). The incident wavelength was $\lambda=5.12 \AA$, providing an instrumental resolution of $50 \mu \mathrm{eV}$ at the elastic line. The single crystal was oriented with (110)(001) as the horizontal reciprocal plane, wrapped in a copper foil (to ensure proper thermalization) and placed in a dilution insert inside a cryostat [31]. Special care was taken to ensure proper thermalization of the sample, and the experiment was started after a 6-hour wait at base temperature $(50 \mathrm{mK})$. Data were recorded between $50 \mathrm{mK}$ and $5 \mathrm{~K}$. To get the full data set as a function of $\mathbf{Q}$ and $E$, the sample was rotated in steps of 1 degree and the counting time was about 10 minutes per sample position. The data were then processed to subtract a constant background and to convert the time of flight, sample rotation, and scattering angle into energy transfer and $\mathbf{Q}$ wave vectors (see Appendix A).

\section{RESULTS}

\section{A. Magnetic structure}

In TbGG, because of the orthorhombic $\left(D_{2}\right)$ electric field on the $24 c$ Wyckoff site of the $I a \overline{3} d$ garnet crystal structure [32], the degeneracy of the ${ }^{7} F_{6}$ multiplet of the free $\mathrm{Tb}^{3+}$ ion is completely lifted into thirteen single states [22]. The lowest energy states are thus two closely spaced singlets. As already mentioned, TbGG is, however, reported to order magnetically at $T_{N} \approx 290 \mathrm{mK}[23,24]$. The observed antiferromagnetic structure is of the AFA type [23,24,33] [inset of Fig. 2(b)], that is, multiaxial, with the $\mathrm{Tb}^{3+}$ spins forming six sublattices parallel or antiparallel to the $\langle 100\rangle$ crystallographic directions of the cubic unit cell [the magnetic space group being $I a \overline{3} d^{\prime}$ (BNS 230.148)]. This $A F A$ structure is specific to rare-earth garnets with Ising anisotropy and dominant dipolar interactions [23,34-36].

As illustrated in Fig. 2(a), neutron powder diffraction experiments confirm this $A F A$ magnetic order for TbGG. The peculiar temperature evolution of the $\mathrm{Tb}^{3+}$ ordered moment [Fig. 2(b)] is very similar to that reported in [37], with $T_{N} \approx$ $280 \mathrm{mK}$, and with the $\mathrm{Tb}^{3+}$ moment reaching only $\approx 4.5 \mu_{B}$ at $45 \mathrm{mK}$.
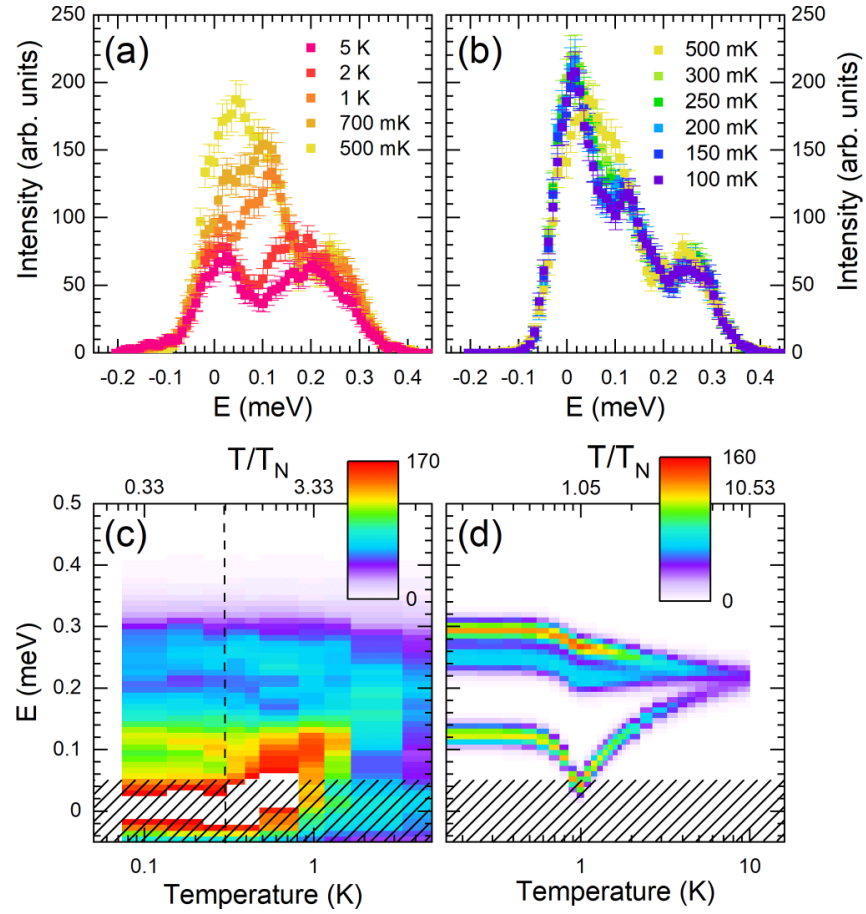

FIG. 3. (a) and (b): Temperature evolution of $E$ scans at constant $Q=(110)$, measured in single-crystal TbGG, above and below $500 \mathrm{mK}$, respectively. (c) Color plot of the same data as (a) and (b) [top $x$ axis: reduced temperature $\left(T / T_{N}\right)$ scale]. The dashed vertical line shows $T_{N}$, below which a strong elastic signal is observed. (d) Corresponding calculation, for the model described in the text, with $x_{\text {dip }}=1$. The shaded area around the elastic line indicates the $50 \mu \mathrm{eV}$ instrumental resolution. The elastic contribution is not shown for clarity in (d).

\section{B. Spin dynamics}

To clarify the formation of this magnetic ground state, the TbGG crystalline electric field (CEF) excitations were studied by INS at $10 \mathrm{~K}$ on both polycrystalline and single-crystal samples. For both, the observed levels are in perfect agreement with a recent and comprehensive neutron scattering study of TbGG by Wawrzynczak et al. [24]. In particular, a very broad signal is seen for an energy transfer $E$ ranging between 4 and $7 \mathrm{meV}$, which is attributed to 4 distinct CEF modes [24], alongside a very low-energy mode around $0.2 \mathrm{meV}$ [see Fig. 3(a) at $5 \mathrm{~K}$ ]. Note that this CEF scheme confirms the strong Ising anisotropy of the $\mathrm{Tb}^{3+}$ ion in TbGG [24], as well as the two-singlet ground state, made of two closely spaced singlets $(\Delta \approx 0.2 \mathrm{meV})$. More details can be found in Appendix B.

Low-energy excitations $(E \leqslant 2 \mathrm{meV})$ were further studied below $1 \mathrm{~K}$ on the TbGG single crystal to investigate the behavior of this two-singlet system through and below $T_{N}$ ( $T_{N}$ being about $300 \mathrm{mK}$ in the TbGG single crystal). As already observed experimentally on polycrystalline TbGG [33], the low-energy CEF level around $0.2 \mathrm{meV}$ becomes dispersive when $T$ approaches $T_{N}$ [38], i.e., when correlations grow, which indicates that the high-temperature single-ion 
excitation has been replaced by a (propagating) exciton. The evolution with temperature of energy scans at the magnetic Bragg $Q=(110)$ is illustrated in Figs. 3(a) and 3(b), with the corresponding color plot in Fig. 3(c). It shows that, far above $T_{N}$, a single CEF mode is observed at $0.2 \mathrm{meV}$. This mode splits into two branches with decreasing temperature, one branch softening down to the elastic line (within the instrumental resolution) at $T_{N}$, and the other branch hardening to reach $0.25 \mathrm{meV}$ at $100 \mathrm{mK}$. Experimentally, it is not possible to affirm whether there is a complete softening or not of the exciton at the transition; if there is a gap, however, it has to be less than $50 \mu \mathrm{eV}$.

This softening fits the theoretical picture of induced magnetism, as explained in Refs. [23,25,26]. If one labels the two singlets as $|1\rangle$ and $|2\rangle$, the singlet nature imposes $\left\langle 1\left|\mathbf{J}_{i}\right| 1\right\rangle=$ $\left\langle 2\left|\mathbf{J}_{i}\right| 2\right\rangle \equiv 0$ ( $\mathbf{J}_{i}$ is the total angular momentum), with however $\left\langle 1\left|\mathbf{J}_{i}\right| 2\right\rangle$ nonzero. If magnetic interactions overcome the energy separation $\Delta$ between the singlets, the system can take advantage of the finite $\left\langle 1\left|\mathbf{J}_{i}\right| 2\right\rangle$ matrix element to mix the $|1\rangle$ and $|2\rangle$ wave functions, to form a magnetic ground state (see also Appendix C). This can be modeled quantitatively using the following Hamiltonian:

$$
\mathcal{H}=\mathcal{H}_{\mathrm{CEF}}+\sum_{\langle i, j\rangle} \sum_{a, b=x, y, z} \mathcal{K}_{i, j}^{a b} \mathbf{J}_{i}^{a} \mathbf{J}_{j}^{b},
$$

where $\mathcal{K}_{i, j}$ describes the interaction tensor between the components of $\mathbf{J}^{a=x, y, z}$ at $\mathrm{Tb}^{3+}$ sites $i$ and $j$ of the hyperkagome network of TbGG. In what follows, it is assumed that $\mathcal{K}_{i, j}$ identifies with the dipolar interaction term $\mathcal{D}_{i, j}$, which is tuned artificially by a parameter labeled $x_{\text {dip }}, \mathcal{K}_{i, j}=x_{\text {dip }} \mathcal{D}_{i, j}$,

$$
\mathcal{K}_{i, j}^{a, b}=x_{\operatorname{dip}} \frac{\mu_{o}}{4 \pi} \frac{\left(g_{J} \mu_{B}\right)^{2}}{r_{i, j}^{3}}\left(\delta_{a, b}-3 \frac{r_{i, j}^{a} r_{i, j}^{b}}{r_{i, j}^{2}}\right),
$$

and is truncated to nearest neighbors. $r_{i j}$ is the vector joining sites $i$ and $j$. Using the nearest-neighbor distance $d_{n n}=$ $\frac{a}{4} \sqrt{3 / 2}$ ( $a$ is the cubic lattice spacing, $d_{n n} \approx 3.78 \AA$ in TbGG [27]), this gives $\mathcal{D} \approx 0.02 \mathrm{~K}$. $\mathcal{H}_{\mathrm{CEF}}$ is based on the parameters proposed by [24] to describe TbGG. There is no magnetic exchange term.

On the basis of this minimalist Hamiltonian, spin dynamics was then calculated in the framework of the RPA approximation (see also Appendix D 1). $S(Q, E)$ maps of the exciton dispersion along [ $h h 0]$, calculated at various temperatures using this model Hamiltonian, are given in Fig. 4. Cooling from $10 \mathrm{~K}$, calculations clearly show that the CEF mode at about $0.2 \mathrm{meV}$ becomes dispersing, being now rather an exciton than a local crystal field excitation. Further decreasing temperature, several modes can be identified, one of them softening progressively before condensing at the Néel temperature. In the ordered phase, the latter hardens again, as a result of the strong anisotropic nature of the dipolar interaction.

For easier comparison with the experimental data of Fig. 3(c), Fig. 3(d) displays the temperature dependence of the calculated excitation spectrum at $Q=(110)$, as extracted from Fig. 4. The model accounts very well for the softening of the exciton at $T_{N}$ which is seen experimentally, and gives a very reasonable value for the energy of the dispersive excitons below $T_{N}$. It predicts another mode of weak amplitude, at $\approx 0.3 \mathrm{meV}$, which is not seen in the experimental data,

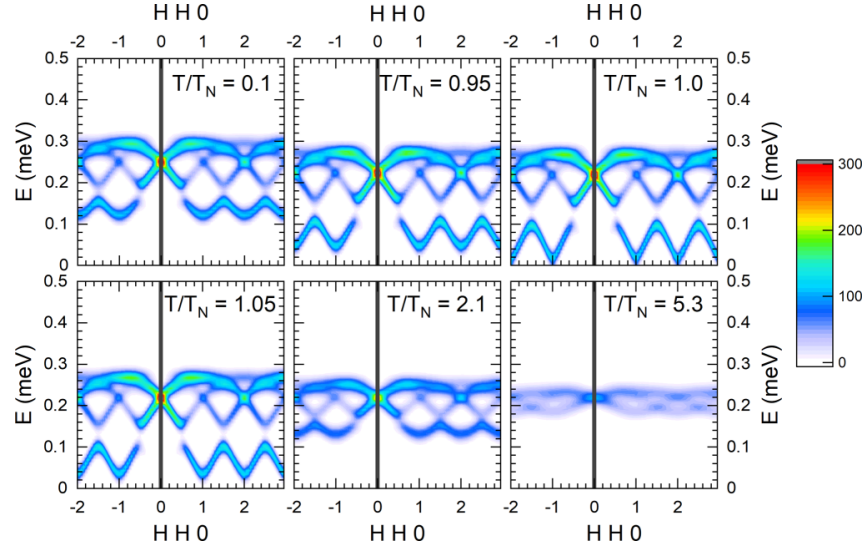

FIG. 4. Reduced temperature evolution of the calculated $S(Q, E)$ spectra of TbGG, along [ $h h 0](E \leqslant 2 \mathrm{meV})$. Calculations are carried out using the Hamiltonian described in the main text, with the CEF parameters of [24], a dipolar interaction parameter $x_{\text {dip }}=1$, and no magnetic exchange.

presumably owing to the limited resolution and/or to the low intensity of the mode itself.

The temperature evolution of the group of CEF levels at higher energies, between 4 and $7 \mathrm{meV}$, is shown in Fig. 5 . In this energy range, the excitonic character of the CEF excitations is not observed anymore, since the strength of the magnetic interactions is negligible compared with the bare energies of the CEF levels, and only a slight change of the levels' energy positions is seen below $T_{N}$.

The moment at saturation calculated from the admixture of states is $M_{s}=4 \mu_{B}$, in very good agreement with the ordered moment value at $45 \mathrm{mK}$ refined from the diffraction data. It is also noteworthy that there is no need to introduce hyperfine coupling into the Hamiltonian to drive the magnetic order, an issue which was still open in TbGG to date [39], and in contrast, for instance, to $\mathrm{Ho}_{3} \mathrm{Ga}_{5} \mathrm{O}_{12}$, another non-Kramers garnet [40]. The main discrepancies between this model and the experimental results are the value of $T_{N}$, calculated to be $950 \mathrm{mK}$ (against $280 \mathrm{mK}$ experimentally), and the shape of the ordered moment temperature evolution, which reaches saturation below $T / T_{N}=0.5$, that is, much faster than experimental observations [Fig. 2(b)]. Discrepancies between the observed and calculated intensity of the modes around

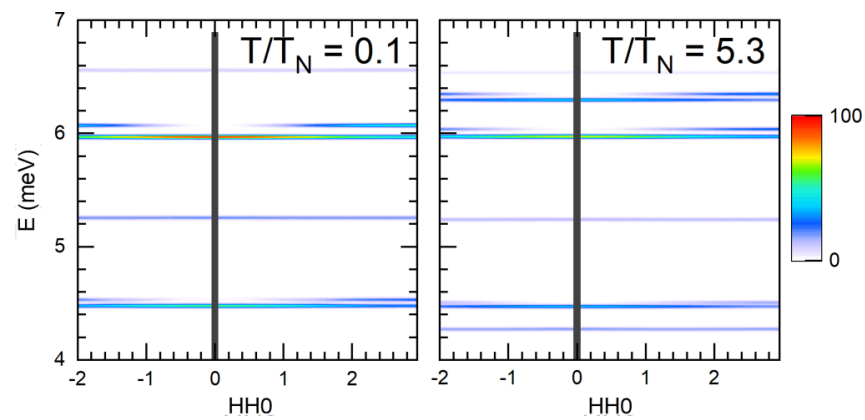

FIG. 5. TbGG $S(Q, E)$ spectra along [ $h h 0]$, calculated below $\left(T / T_{N}=0.1\right)$ and above $\left(T / T_{N}=5.3\right) T_{N}$, shown between 4 and $7 \mathrm{meV}$. 

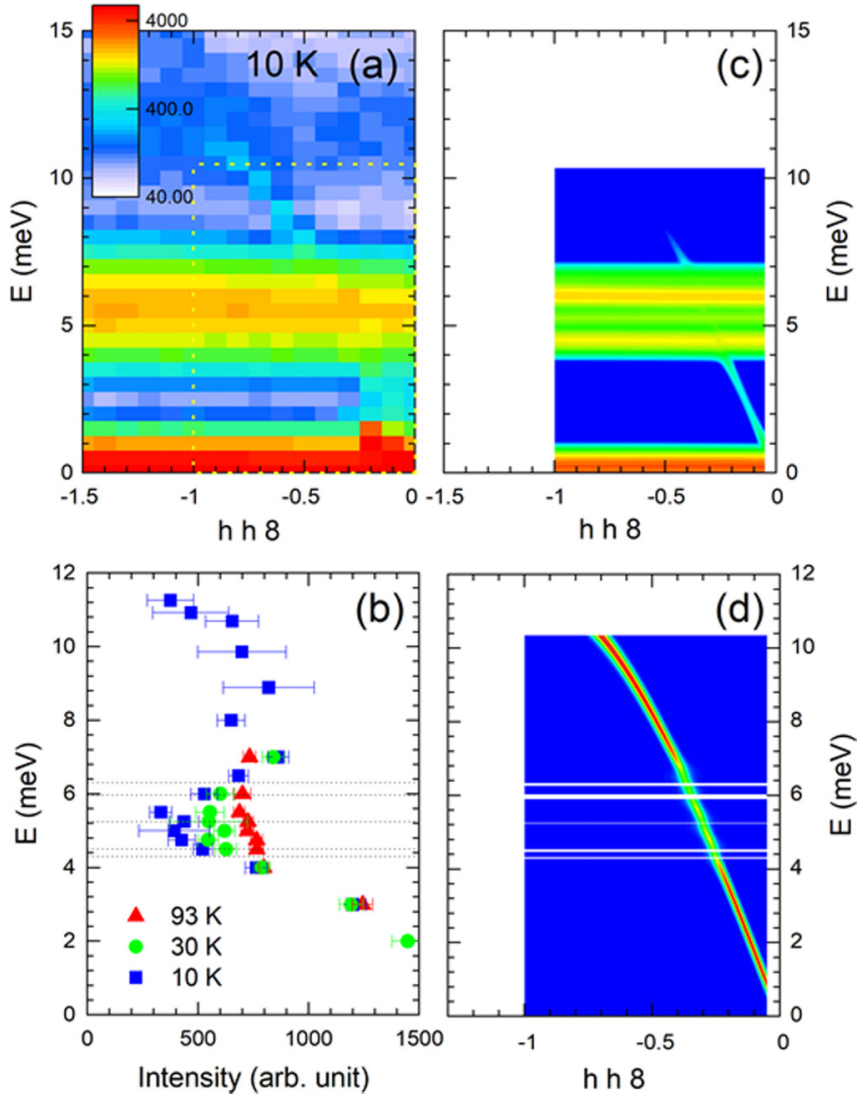

FIG. 6. (a) Experimental $S(Q, E)$ at $10 \mathrm{~K}$ along [hh8]. (b) Fitting of the phonon branch intensity versus $E$, at different temperatures. (c) and (d): Calculations performed assuming a magnetoelastic coupling of $0.3 \mathrm{~K}$ with a single acoustic phonon branch. (c) shows the total neutron intensity (magnetic and phononic), while (d) shows the phononic contribution only, to emphasize the variations of the intensity of the phonon branch as it crosses the different CEF levels (pictured by horizontal lines).

$0.25 \mathrm{meV}$ can also be seen when comparing Figs. 3(c) and $3(d)$. These are indications that mean-field theory with the chosen Hamiltonian, although it leads to an overall correct description of TbGG, is likely not accurate enough to describe finer details, and that a more complex Hamiltonian, or a more sophisticated theoretical approach, is necessary.

\section{Lattice dynamics}

To go further into the understanding of TbGG magnetoelastic properties, phonon dynamics was investigated by INS at $10 \mathrm{~K}$; experimentally this study was performed at rather large $Q$ values, to increase the phonon form factor, and in an energy range up to $20 \mathrm{meV}$. Several tests were performed around different zone centers, but $Q=(008)$ was eventually chosen to enhance as much as possible the phonon cross section. The $(Q, E)$ space was then mapped out, probing both the longitudinal and transverse acoustic modes stemming from the corresponding Bragg peak. The energy resolution did not allow one to study the $0.2 \mathrm{meV}$ level, the latter being buried into the elastic line. The results are illustrated in Fig. 6(a), which shows the INS spectrum $S(Q, E)$ of TbGG built from constant- $E Q$ scans, mapped along [ $h h 8]$. A steep transverse acoustic phonon branch, propagating along [hh0] and polarized along [001], is clearly visible, linearly stemming from the (008) Bragg position, and crossing the group of CEF levels located between 4 and $7 \mathrm{meV}$. The intensity of those modes is still strong, despite the large wave vectors. An additional scattering is visible around $12 \mathrm{meV}$, a feature which is seen also on the polycrystalline TbGG $S(Q, E)$ spectrum at $5 \mathrm{~K}$. It is tentatively attributed to an optical phonon mode, as its intensity increases with $Q$.

The intensity of the phonon mode was fitted using the following equation:

$$
\begin{aligned}
I(E, Q=(h h 8))= & c+\left(1+\frac{1}{e^{E / k_{B} T}-1}\right) \\
& \times A(E)\left[g\left(h, h_{E}\right)+g\left(h,-h_{E}\right)\right],
\end{aligned}
$$

where $c$ is an effective flat background and $g\left(h, h_{E}\right)=$ $e^{-4 \log (2)\left(h-h_{E}\right)^{2} / \gamma^{2}}$ is a Gaussian profile centered on $h_{E}(\gamma$ is its FWHM). This analysis, repeated at 4 , 30, and $93 \mathrm{~K}$, leads to the results displayed in Fig. 6(b). Strikingly, the $A(E)$ amplitude shows a dip when crossing the 4-7 meV CEF levels, when a classical $1 / E$ behavior would have been expected. Furthermore, this dip progressively fills up with increasing temperature, to become barely observable at $100 \mathrm{~K}$. The same fitting, repeated for the longitudinal phonon mode propagating and polarized along [001], did not reveal the same anomaly.

\section{RPA modeling}

To qualitatively interpret these measurements, it seems straightforward to add a magnetoelastic coupling term to Eq. (1). The simplest way is to couple an atomic displacement to the quadrupolar moments of the $\mathrm{Tb} 4 f$ electronic distribution. The latter writes $\mathbf{Q}_{i}=\sum_{a, b} q_{a b} X_{i}^{a b}$, where $X_{i}^{a b}=$ $|a, i\rangle\langle b, i|$ denote the transitions between the CEF eigenstates $|a\rangle$ and $|b\rangle$ at site $i$, and where the $q_{a b}$ coefficients depend on the details of $\mathcal{H}_{\mathrm{CEF}}$ (see Appendix B and [24]). The atomic displacements write as $\left(a_{-k s}^{+}+a_{k, s}\right)$ in terms of phonon creation and annihilation operators ( $s$ labels the band index, $k$ the momentum, and $\Omega_{k, s}$ the phonon energy), so that the new Hamiltonian becomes

$$
\begin{aligned}
\mathcal{H}^{\prime}= & \mathcal{H}+\sum_{k, s} \Omega_{k, s} a_{k s}^{+} a_{k, s} \\
& +\sum_{k, s, i} v_{i, k, s}\left(a_{-k s}^{+}+a_{k, s}\right)\left(X_{i}^{a b}+X_{i}^{b a}\right) .
\end{aligned}
$$

It includes a direct coupling $v_{i, k, s}$ between phonons and electronic transitions, which, because of resonant coupling, leads to hybrid magnetoelastic modes. This interaction was thus incorporated into the RPA code used previously (Appendix D 1 ). In the calculations, a simple transverse acoustic mode $\Omega_{k, s}$ is considered and an identical effective coupling $v$ is assumed between this mode and the CEF transitions. Figures 6(c) and 6(d) display the calculated spin and lattice contributions to the neutron cross section, respectively, and evidence the expected mixing of the bare phonon and CEF excitations. Owing to the proximity of several CEF modes in the 4-7 meV range, this hybridization manifests itself as a decrease of the phonon spectral weight, in agreement with what is observed experi- 
mentally [Fig. 6(b)]. To reproduce the experimental data, the strength of the effective coupling was adjusted to $0.3 \mathrm{~K}$.

\section{DISCUSSION}

From an analysis of the temperature and field dependence of the thermal conductivity of TbGG, it had been previously inferred [20] that including in the data modeling resonant scattering of phonons with CEF transition frequencies corresponding to transitions $X^{12}$ and $X^{34}$ was giving a satisfactory agreement (with the addition of resonant scattering from a few impurities). Scattering from the $X^{34}$ was also found accountable in a broad temperature range, from $8 \mathrm{~K}$ to $200 \mathrm{~K}$. The present study goes further, and now provides microscopic evidence for the coupling between acoustic phonons and at least four of the $\mathrm{Tb}^{3+}$ six lowest excited states.

With regard to the phonon Hall effect at $5 \mathrm{~K}$, the mean free path of phonons with frequencies close to the resonant frequencies of the $X^{12}$ and $X^{34}$ transitions is on the order of the phonon wavelength; when the splitting between levels becomes too large, like when a field is applied in a specific direction such as [111], the resonant scattering rate decreases and thermal conductivity peaks. Moreover, the magnetoelastic interaction is also expected to change the topology of the phonon bands, giving rise to "anomalous" velocities and thus to off-diagonal heat conductivity. Because of the field dependence of the CEF, those bands will strongly depend on the field amplitude, leading to a significant thermal Hall effect. Furthermore, owing to the strong Ising character of $\mathrm{Tb}^{3+}$ on its site, this splitting differs depending on the orientation of the local Ising axis with respect to the applied magnetic field.

While a detailed microscopic derivation of the magnetoelastic Hamiltonian would likely give a more reliable value of the coupling constants $v$, it is important to mention that $\mathcal{H}^{\prime}$ describes, at low energies, a two-level system $(|1\rangle$ and $|2\rangle)$ coupled to a bath of harmonic oscillators, a very difficult problem relevant to many areas of physics, from quantum decoherence to polaron formation $[41,42]$. The physical effect of the coupling is the "polarization" of a given state by the other one: $|1\rangle$ (resp. $|2\rangle$ ) becomes dressed by an admixture of $|2\rangle$ (resp. |1 $\rangle$ ), surrounded by a cloud of phonons. Within the mean-field framework, this may translate into the formation of a static distorted structure if the coupling $v$ overcomes the energy separation $\Delta$. This is indeed the case in the above RPA calculations, which yields $v \approx 0.3 \mathrm{~K}$, a very large value, likely an artifact of a too crude approximation, neglecting zero point fluctuations especially. It remains that the coupling to the phonon bath results in a mixing of the $|1\rangle$ and $|2\rangle$ states, leading to new electronic states which can be written, for the sake of illustration, $\left|1^{\prime}\right\rangle=u|1\rangle+v|2\rangle$ and $\left|2^{\prime}\right\rangle=-v|1\rangle+$ $u|2\rangle$. Interestingly this mechanism may confer an emergent fluctuating magnetic moment to the new electronic states $\left\langle 1^{\prime}\left|\mathbf{J}_{i}\right| 1^{\prime}\right\rangle=-\left\langle 2^{\prime}\left|\mathbf{J}_{i}\right| 2^{\prime}\right\rangle=2 u v\left\langle 1\left|\mathbf{J}_{i}\right| 2\right\rangle$, which could prevent the formation of any static order, or compete with the dipolar interaction induced mechanism. In the case of TbGG, such a competition could be the reason behind the discrepancy between the experimental $T_{N}$ of $280 \mathrm{mK}$ and the theoretical one of $950 \mathrm{mK}$ calculated considering a bare singlet-singlet model, that is, decoupled from the phonon bath. Within this scenario, magnetoelastic coupling delays the $A F A$ magnetic ordering, possibly explaining the peculiar temperature evolution of the $\mathrm{Tb}^{3+}$ ordered magnetic moment below $T_{N}$ as well.

Such physics may be relevant to other $\mathrm{Tb}$ compounds, such as the pyrochlores $\mathrm{Tb}_{2} \mathrm{Ti}_{2} \mathrm{O}_{7}$ or $\mathrm{Tb}_{2} \mathrm{Sn}_{2} \mathrm{O}_{7}$ [43-45]. In both, a similar coupling between the quadrupolar moments of the electronic density and the lattice degrees of freedom is also at play [46-50]. In contrast with TbGG, the multiplet degeneracy is not totally lifted, so that the low-energy CEF scheme is described by a degenerate Ising magnetic doublet $m=\left\langle 1\left|\mathbf{J}_{i}\right| 1\right\rangle=-\left\langle 2\left|\mathbf{J}_{i}\right| 2\right\rangle \neq 0$ and characterized by a strictly zero matrix element $\left\langle 1\left|\mathbf{J}_{i}\right| 2\right\rangle \equiv 0$ [51,52]. However, the coupling to the phonon bath may still favor the formation of perturbed states made of a mixing of $|1\rangle$ and $|2\rangle$. This would not only confer a fluctuating reduced magnetic moment to the new electronic states $\left\langle 1^{\prime}\left|\mathbf{J}_{i}\right| 1^{\prime}\right\rangle=-\left\langle 2^{\prime}\left|\mathbf{J}_{i}\right| 2^{\prime}\right\rangle=m\left(u^{2}-v^{2}\right)$, but also a fluctuating nonzero matrix element $\left\langle 1^{\prime}\left|\mathbf{J}_{i}\right| 2^{\prime}\right\rangle=$ $2 u v m \neq 0$, with two consequences: (i) to prevent, or compete with, the formation of magnetic long-range order, and (ii) to give rise to low-energy dynamics, taking the form of an exciton built on the new electronic states. The facts that $\mathrm{Tb}_{2} \mathrm{Ti}_{2} \mathrm{O}_{7}$ remains disordered down to $50 \mathrm{mK}$ while INS data provide evidence for very low energy spin dynamics both in $\mathrm{Tb}_{2} \mathrm{Ti}_{2} \mathrm{O}_{7}$ [47] and $\mathrm{Tb}_{2} \mathrm{Sn}_{2} \mathrm{O}_{7}$ [45] are two features which have eluded proper understanding up to now, but which could be understood in this framework.

\section{CONCLUSIONS}

Neutron scattering measurements combined with RPA calculations have shed light on the intriguing properties of $\mathrm{Tb}_{3} \mathrm{Ga}_{5} \mathrm{O}_{12}$. They confirm the picture of TbGG as an archetypal two-singlet system, in which dipolar interactions are strong enough to mix the two singlets and lead to a magnetic state. Magnetoelastic coupling has a major role in addition, resulting in the formation of magnetoelastic hybrid modes with arguably direct consequences on the phonon heat-carrying properties including PTHE. Whether this coupling along with lattice zero point motion also influences low-energy dynamics still remains to be studied. More generally, our results suggest that eigenstates coupled with harmonic oscillators could be a pertinent physical approach to $\mathrm{Tb}$ systems.

\section{ACKNOWLEDGMENTS}

S.P. and F.D. acknowledge financial support from the French Federation of Neutron Scattering (2FDN). This work was supported by "Investissements d'Avenir" LabEx PALM (ANR-10-LABX-0039-PALM, 1Dmag project).

\section{APPENDIX A: TOF DATA REDUCTION}

Reduction of the TOF data from IN6-SHARP was performed using a homemade code which applies a transformation which relates the Cartesian coordinates of the instrument to the rotated reciprocal lattice coordinates. The procedure assumes that two reciprocal lattice vectors $\mathbf{u}$ and $\mathbf{v}$ are identified, and that their Cartesian coordinates $\tilde{\mathbf{u}}$ and $\tilde{\mathbf{v}}$ (along with 
$\tilde{\mathbf{w}}=\tilde{\mathbf{u}} \times \tilde{\mathbf{v}})$ are known. The transformation matrix thus writes

$$
M=\left(\begin{array}{ccc}
\tilde{u}_{x} & \tilde{v}_{x} & \tilde{w}_{x} \\
\tilde{u}_{y} & \tilde{v}_{y} & \tilde{w}_{y} \\
\tilde{u}_{z} & \tilde{v}_{z} & \tilde{w}_{z}
\end{array}\right) .
$$

For instance, if $\tilde{\mathbf{u}}$ and $\tilde{\mathbf{v}}$ are defined with Euler angles $\alpha$ and $\beta$ (being the azimuth), those coordinates write

$$
\begin{aligned}
& \tilde{\mathbf{u}}=\|\tilde{u}\|\left(\begin{array}{c}
\cos \alpha_{u} \cos \beta_{u} \\
\sin \alpha_{u} \cos \beta_{u} \\
\sin \beta_{u}
\end{array}\right), \\
& \tilde{\mathbf{v}}=\|\tilde{v}\|\left(\begin{array}{c}
\cos \alpha_{v} \cos \beta_{v} \\
\sin \alpha_{v} \cos \beta_{v} \\
\sin \beta_{v}
\end{array}\right), \\
& \tilde{\mathbf{w}}=\tilde{\mathbf{u}} \times \tilde{\mathbf{v}} .
\end{aligned}
$$

Any wave vector written as $\mathbf{Q}=\lambda \mathbf{u}+\mu \mathbf{v}+\nu \mathbf{w}$ has thus Cartesian coordinates given by

$$
\tilde{\mathbf{Q}}=M \cdot \mathbf{Q} \text {. }
$$

During the experiment, the sample is rotated step by step around the vertical axis by the angle $\phi_{k}$. This rotation depends on an offset $\Phi$, which encodes how the sample was positioned on the dilution stick. Accordingly, we have to consider, for each $\phi_{k}$, a $M_{k}$ matrix, consisting of the collection of the three vectors $\tilde{u}, \tilde{v}, \tilde{w}$ rotated around the vertical axis by the angle $\phi_{k}+\Phi ;$ hence,

$$
\mathbf{Q}=M_{k}^{-1} \cdot \tilde{\mathbf{Q}} .
$$

In these conditions, the recorded intensity $I\left(E, \theta, \phi=\phi_{k}\right)$ is associated with a scattering process with the incoming wave vector $k_{i}=2 \pi / \lambda$, the outgoing wave vector

$$
k_{f}=\sqrt{k_{i}^{2}-E / a}, \quad a \approx 2.0 \mathrm{meV} \AA^{2},
$$

and the scattering wave vector $\mathbf{Q}$ defined by its Cartesian coordinates $\tilde{\mathbf{Q}}$ :

$$
\begin{aligned}
& \tilde{Q}_{x}=k_{i}-k_{f} \cos \theta, \\
& \tilde{Q}_{y}=-k_{f} \sin \theta, \\
& \tilde{Q}_{z}=0 .
\end{aligned}
$$

The reasoning is here restricted to the horizontal plane, but a generalization is straightforward if considering the $z$ axis. Back to the physical reciprocal lattice, the wave vector writes

$$
\mathbf{Q}=M_{k}^{-1} \cdot \tilde{\mathbf{Q}} .
$$

It remains to integrate the recorded intensity on a user-defined $(\mathbf{Q}, E)$ grid and determine the offset $\Phi$ while identifying the Bragg peaks.

\section{APPENDIX B: $\mathrm{Tb}_{3} \mathrm{Ga}_{5} \mathrm{O}_{12}$ CRYSTAL ELECTRIC FIELD CHARACTERIZATION}

INS measurements carried out on $2 \mathrm{~T}$ and IN6 confirm existing experimental data, as illustrated in Fig. 7, for instance. We thus did not attempt to determine the crystal field scheme and used the results of Ref. [24] instead. The energy levels are given in Table I and the lowest energy wave functions are

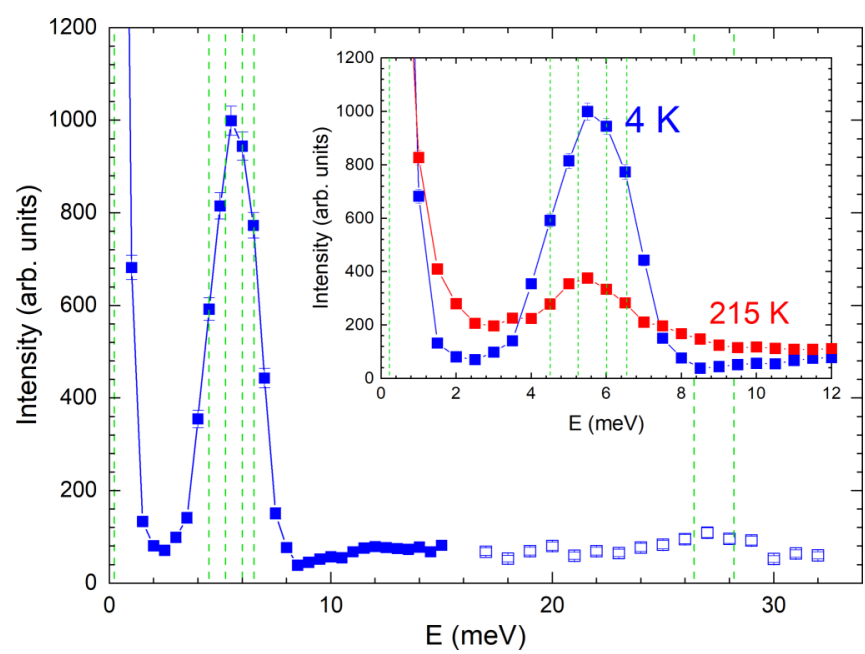

FIG. 7. $E$ scan at constant $Q=2.2 \AA^{-1}$ of the powder neutron scattering spectrum of TbGG at $10 \mathrm{~K}$. Green vertical lines indicate the positions of the CEF excitations calculated by [24]. The four CEF levels are not resolved because of the $\Delta E \approx 1.2 \mathrm{meV}$ resolution of the experimental setup. Inset: Corresponding temperature evolution.

given by

$$
\begin{aligned}
|1\rangle \approx & -0.09\left|\mathbf{J}_{z}= \pm 5\right\rangle+0.70\left|\mathbf{J}_{z}= \pm 1\right\rangle, \\
|2\rangle \approx-0.18 \mid \mathbf{J}_{z} & = \pm 6\rangle-0.12\left|\mathbf{J}_{z}= \pm 4\right\rangle+0.32\left|\mathbf{J}_{z}= \pm 2\right\rangle \\
& +0.83\left|\mathbf{J}_{z}=0\right\rangle .
\end{aligned}
$$

As in rare-earth pyrochlores, it is convenient to work in local bases. In the garnet structure, the subset of magnetic ions is composed of $2 \times 12$ atoms, which form two intertwined hyperkagome networks. The $24 \mathrm{c}$ site occupied by magnetic ions in the Ia $\overline{3} d$ space group has $D_{2}$ orthorhombic local symmetry; the main axis is one of the cubic axes. Those local bases are identical for the two networks. Each block of 12 ions is constructed on two equivalent groups of 6 atoms, separated by a translation $(1 / 2,1 / 2,1 / 2)$. Atomic positions, numbering, and local CEF axes are given in Table II.

TABLE I. TbGG energy levels determined in Ref. [24]. The quantization axis is given in Table II.

\begin{tabular}{rc}
\hline \hline$n$ & Energy levels $(\mathrm{meV})$ \\
\hline 2 & 0.21 \\
3 & 4.48 \\
4 & 5.24 \\
5 & 6.01 \\
6 & 6.54 \\
7 & 26.41 \\
8 & 28.28 \\
9 & 34.27 \\
10 & 34.75 \\
11 & 35.65 \\
12 & 36.39 \\
13 & 38.22 \\
\hline
\end{tabular}


TABLE II. Rare-earth atom labels, positions, and local CEF axes in the garnet $I a \overline{3} d$ crystal structure.

\begin{tabular}{|c|c|c|c|c|}
\hline$N$ & Position & $y$ axis & $z$ axis & $x$ axis \\
\hline 1 & $\left(\frac{1}{8}, 0, \frac{1}{4}\right)$ & $(0,1, \overline{1})$ & $(0,1,1)$ & $(1,0,0)$ \\
\hline 2 & $\left(\frac{3}{8}, 0, \frac{3}{4}\right)$ & $(0, \overline{1}, \overline{1})$ & $(0, \overline{1}, 1)$ & $(\overline{1}, 0,0)$ \\
\hline 3 & $\left(\frac{1}{4}, \frac{1}{8}, 0\right)$ & $(\overline{1}, 0,1)$ & $(1,0,1)$ & $(0,1,0)$ \\
\hline 4 & $\left(\frac{3}{4}, \frac{3}{8}, 0\right)$ & $(\overline{1}, 0, \overline{1})$ & $(1,0, \overline{1})$ & $(0, \overline{1}, 0)$ \\
\hline 5 & $\left(0, \frac{1}{4}, \frac{1}{8}\right)$ & $(1, \overline{1}, 0)$ & $(1,1,0)$ & $(0,0,1)$ \\
\hline 6 & $\left(0, \frac{3}{4}, \frac{3}{8}\right)$ & $(\overline{1}, \overline{1}, 0)$ & $(\overline{1}, 1,0)$ & $(0,0, \overline{1})$ \\
\hline 7 & $\left(\frac{5}{8}, \frac{1}{2}, \frac{3}{4}\right)$ & $(0,1, \overline{1})$ & $(0,1,1)$ & $(1,0,0)$ \\
\hline 8 & $\left(\frac{7}{8}, \frac{1}{2}, \frac{1}{4}\right)$ & $(0, \overline{1}, \overline{1})$ & $(0, \overline{1}, 1)$ & $(\overline{1}, 0,0)$ \\
\hline 9 & $\left(\frac{3}{4}, \frac{5}{8}, \frac{1}{2}\right)$ & $(\overline{1}, 0,1)$ & $(1,0,1)$ & $(0,1,0)$ \\
\hline 10 & $\left(\frac{1}{4}, \frac{7}{8}, \frac{1}{2}\right)$ & $(\overline{1}, 0, \overline{1})$ & $(1,0, \overline{1})$ & $(0, \overline{1}, 0)$ \\
\hline 11 & $\left(\frac{1}{2}, \frac{3}{4}, \frac{5}{8}\right)$ & $(1, \overline{1}, 0)$ & $(1,1,0)$ & $(0,0,1)$ \\
\hline 12 & $\left(\frac{1}{2}, \frac{1}{4}, \frac{7}{8}\right)$ & $(\overline{1}, \overline{1}, 0)$ & $(\overline{1}, 1,0)$ & $(0,0, \overline{1})$ \\
\hline 13 & $\left(\frac{7}{8}, 0, \frac{3}{4}\right)$ & $(0,1, \overline{1})$ & $(0,1,1)$ & $(1,0,0)$ \\
\hline 14 & $\left(\frac{5}{8}, 0, \frac{1}{4}\right)$ & $(0, \overline{1}, \overline{1})$ & $(0, \overline{1}, 1)$ & $(\overline{1}, 0,0)$ \\
\hline 15 & $\left(\frac{3}{4}, \frac{7}{8}, 0\right)$ & $(\overline{1}, 0,1)$ & $(1,0,1)$ & $(0,1,0)$ \\
\hline 16 & $\left(\frac{1}{4}, \frac{5}{8}, 0\right)$ & $(\overline{1}, 0, \overline{1})$ & $(1,0, \overline{1})$ & $(0, \overline{1}, 0)$ \\
\hline 17 & $\left(0, \frac{3}{4}, \frac{7}{8}\right)$ & $(1, \overline{1}, 0)$ & $(1,1,0)$ & $(0,0,1)$ \\
\hline 18 & $\left(0, \frac{1}{4}, \frac{5}{8}\right)$ & $(\overline{1}, \overline{1}, 0)$ & $(\overline{1}, 1,0)$ & $(0,0, \overline{1})$ \\
\hline 19 & $\left(\frac{3}{8}, \frac{1}{2}, \frac{1}{4}\right)$ & $(0,1, \overline{1})$ & $(0,1,1)$ & $(1,0,0)$ \\
\hline 20 & $\left(\frac{1}{8}, \frac{1}{2}, \frac{3}{4}\right)$ & $(0, \overline{1}, \overline{1})$ & $(0, \overline{1}, 1)$ & $(\overline{1}, 0,0)$ \\
\hline 21 & $\left(\frac{1}{4}, \frac{3}{8}, \frac{1}{2}\right)$ & $(\overline{1}, 0,1)$ & $(1,0,1)$ & $(0,1,0)$ \\
\hline 22 & $\left(\frac{3}{4}, \frac{1}{8}, \frac{1}{2}\right)$ & $(\overline{1}, 0, \overline{1})$ & $(1,0, \overline{1})$ & $(0, \overline{1}, 0)$ \\
\hline 23 & $\left(\frac{1}{2}, \frac{1}{4}, \frac{3}{8}\right)$ & $(1, \overline{1}, 0)$ & $(1,1,0)$ & $(0,0,1)$ \\
\hline 24 & $\left(\frac{1}{2}, \frac{3}{4}, \frac{1}{8}\right)$ & $(\overline{1}, \overline{1}, 0)$ & $(\overline{1}, 1,0)$ & $(0,0, \overline{1})$ \\
\hline
\end{tabular}

To investigate the coupling between quadrupolar moments and phonons, it is useful to evaluate the matrix of the corresponding operators in the basis formed by the eigenvectors. For the sake of simplicity, we restrict ourselves to the 6 lowest energy states. Interestingly, the largest matrix elements occur for transitions $X^{16}$ (mediated by $\left.\mathbf{O}_{x y}\right), X^{23}$ and $X^{25}\left(\mathbf{O}_{x y}\right)$, and $X^{24}\left(\mathbf{O}_{20}\right)$. A relatively smaller coupling exists for $X^{12}$ and mediated by $\mathbf{O}_{y z}$. This indicates that at $10 \mathrm{~K}$, the 6 lowest energy states are potentially coupled to phonons:

$$
\begin{aligned}
& \mathbf{O}_{20}=\left(\begin{array}{cccccc}
-37.8 & \cdot & \cdot & \cdot & \cdot & \cdot \\
\cdot & -30.8 & \cdot & 25.6 & \cdot & \cdot \\
\cdot & 25.6 & 38.8 & \cdot & 42.7 & \cdot \\
\cdot & \cdot & 59.1 & \cdot & \cdot \\
\cdot & \cdot & 42.7 & \cdot & -1.3 & \cdot \\
\cdot & \cdot & \cdot & \cdot & \cdot & -26.9
\end{array}\right), \\
& \mathbf{O}_{22}=\left(\begin{array}{cccccc}
19.4 & \cdot & \cdot & . & \cdot & \cdot \\
\cdot & 20.3 & . & -3.6 & . & \cdot \\
\cdot & \cdot & 0.5 & . & -2.7 & \cdot \\
\cdot & -3.6 & . & -0.2 & . & \cdot \\
\cdot & \cdot & -2.7 & . & 5.8 & . \\
. & . & . & . & . & 8.4
\end{array}\right) \text {, }
\end{aligned}
$$

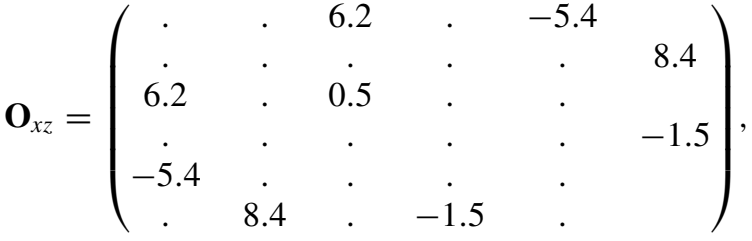

$$
\begin{aligned}
& \mathbf{O}_{x y}=\left(\begin{array}{cccccc}
\cdot & \cdot & \cdot & \cdot & \cdot & 29.7 i \\
\cdot & \dot{16.5 i} & 16.5 i & . & -23.1 i & \cdot \\
\cdot & -16.8 i & \cdot & \cdot \\
\cdot & 23.1 i & -3.8 i & . & 5.0 i & \cdot \\
-29.7 i & . & . & . & . & .
\end{array}\right) \text {, } \\
& \mathbf{O}_{y z}=\left(\begin{array}{cccccc}
\cdot & 1.1 i & \cdot & 2.3 i & \cdot & \cdot \\
-1.1 & \cdot & \cdot & \cdot & \cdot & \cdot \\
-2.3 i & \cdot & \cdot & \cdot & \cdot & 0.1 i \\
\cdot & \cdot & \cdot & \cdot & \cdot & \cdot \\
\cdot & \cdot & -0.1 i & \cdot & \cdot .1 i & -1.1 i
\end{array}\right) .
\end{aligned}
$$

\section{APPENDIX C: SIMPLE CONSIDERATIONS ABOUT TWO-LEVEL SYSTEMS}

\section{Induced magnetization}

In this section, we explain the notion of induced magnetization. For the sake of simplicity, the discussion is restricted to the subspace spanned by the two low-energy singlets (labeled $|1\rangle$ and $|2\rangle$ ) of the full CEF scheme. In this subspace, the CEF Hamiltonian writes

$$
\mathcal{H}_{\mathrm{CEF}}=\left(\begin{array}{cc}
-\Delta / 2 & 0 \\
0 & \Delta / 2
\end{array}\right),
$$

and $\Delta$ is the energy gap between $|1\rangle$ and $|2\rangle$. Since those states are singlets, we have $\langle 1|\mathbf{J}| 1\rangle=\langle 2|\mathbf{J}| 2\rangle \equiv 0$, and we further assume that the transverse matrix elements are Ising-like, with only $\left\langle 1\left|\mathbf{J}_{z}\right| 2\right\rangle=w \neq 0$. The matrix of the magnetic moment thus writes

$$
\mathbf{J}_{x}=0, \quad \mathbf{J}_{y}=0, \quad \mathbf{J}_{z}=\left(\begin{array}{cc}
0 & w \\
w & 0
\end{array}\right) .
$$

As a result, an external magnetic field, which we shall identify later on with a (self-consistent) molecular field, allows for transitions between the two states, yielding

$$
\mathcal{H}=\mathcal{H}_{\mathrm{CEF}}+\mathbf{h} \cdot \mathbf{J}=\left(\begin{array}{cc}
-\Delta / 2 & y \\
y & \Delta / 2
\end{array}\right) .
$$

Here, $y$ stands for $y=h w$. It is straightforward to show that this Hamiltonian is diagonalized in the basis of eigenvectors

$$
\left(\begin{array}{cc}
\cos \theta & -\sin \theta \\
\sin \theta & \cos \theta
\end{array}\right)
$$

with

$$
\tan 2 \theta=-\frac{2 y}{\Delta}
$$

and eigenvalues $\epsilon_{+}$and $\epsilon_{-}$:

$$
\epsilon_{ \pm}= \pm \frac{\sqrt{\Delta^{2}+4 y^{2}}}{2} .
$$




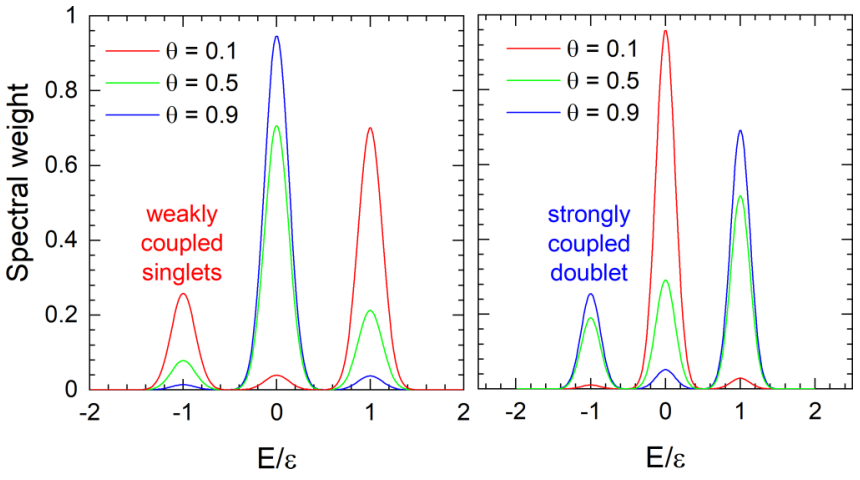

FIG. 8. Neutron spectral weight as a function of energy, for different angles $\theta$, ranging from the weakly coupled $(\theta=0.1)$ to the strongly coupled case $(\theta=0.9)$. The left and right panels show the two-singlet case and the Ising non-Kramers doublet case, respectively.

In this basis, the matrix of the magnetic moment writes

$$
\mathbf{J}_{x}=0, \quad \mathbf{J}_{y}=0, \quad \mathbf{J}_{z}=w\left(\begin{array}{cc}
\sin 2 \theta & \cos 2 \theta \\
\cos 2 \theta & -\sin 2 \theta
\end{array}\right) .
$$

In other words, a soon as $\theta \neq 0$, the new states get a finite magnetic moment and a reduced off-diagonal element:

$$
\begin{aligned}
\left|1^{\prime}\right\rangle & =\cos \theta|1\rangle+\sin \theta|2\rangle, \\
\left|2^{\prime}\right\rangle & =-\sin \theta|1\rangle+\cos \theta|2\rangle, \\
\left\langle 1^{\prime}\left|\mathbf{J}_{z}\right| 1^{\prime}\right\rangle & =w \sin 2 \theta, \\
\left\langle 2^{\prime}\left|\mathbf{J}_{z}\right| 2^{\prime}\right\rangle & =-w \sin 2 \theta, \\
\left\langle 1^{\prime}\left|\mathbf{J}_{z}\right| 2^{\prime}\right\rangle & =w \cos 2 \theta .
\end{aligned}
$$

The averaged magnetization writes

$$
\begin{aligned}
\mathbf{m} & =\frac{1}{Z} \operatorname{Tr}\left(e^{-\mathcal{H} / T} \mathbf{J}\right), \quad Z=e^{-\epsilon_{+} / T}+e^{-\epsilon_{-} / T}, \\
\mathbf{m}_{z} & =-w \frac{2 y}{\sqrt{\Delta^{2}+4 y^{2}}} \tanh \frac{\sqrt{\Delta^{2}+4 y^{2}}}{2 T} .
\end{aligned}
$$

It is interesting to further examine the neutron cross section in this simplified model. Owing to the matrix of the magnetic moment, this quantity is proportional to

$$
\begin{aligned}
I= & (w \sin 2 \theta)^{2} \delta(E) \\
& +\frac{1}{Z}(w \cos 2 \theta)^{2}\left[\delta(E-\epsilon)+e^{-\epsilon / T} \delta(E+\epsilon)\right]
\end{aligned}
$$

and thus shows an elastic contribution along with inelastic peaks at $E= \pm \epsilon$. If the elastic peak is weak, the weight of the inelastic transitions saturates up to $w^{2}$. In contrast, if the elastic peak goes up to $w$, the weight of the inelastic transitions vanishes (see Fig. 8).

At the mean-field level, the magnetic field will be replaced by a molecular field, directly related to the magnetization itself via a coefficient $\mathcal{J}$, which physically describes the strength of the interactions, yielding $h=\mathcal{J} \mathbf{m}_{z}$ hence $y=$ $\mathcal{J} \mathbf{m}_{z} w$. The previous equation is thus a self-consistent equa-

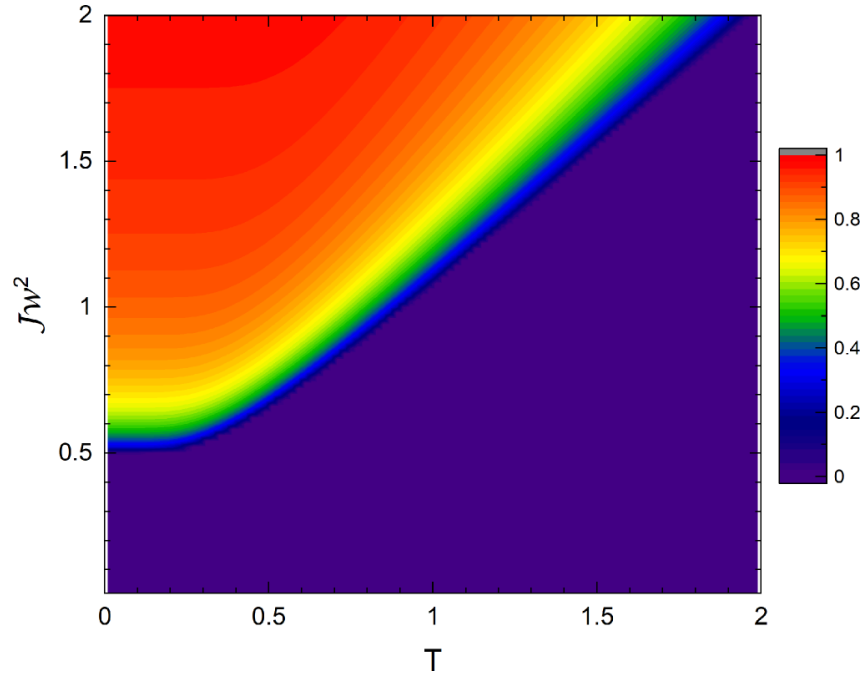

FIG. 9. Mean-field phase diagram of the two-singlet problem. Calculations are carried out with $\Delta=1$. The color scale encodes the value of $y / \mathcal{J} w^{2}$.

tion for $y$ :

$$
y=-y \frac{2 \mathcal{J} w^{2}}{\sqrt{\Delta^{2}+4 y^{2}}} \tanh \frac{\sqrt{\Delta^{2}+4 y^{2}}}{2 T} .
$$

Beyond the trivial solution $y=0$, another solution might exist, depending on temperature and on the actual value of $\mathcal{J}$. At low temperature, one obtains

$$
y \approx \mathcal{J} w^{2} \sqrt{1-\left(\frac{\Delta}{2 \mathcal{J} w^{2}}\right)^{2}} .
$$

As shown in Fig. 9, a magnetic state is thus stabilized (or "induced") if the magnetic energy gain overcomes the energy gap $\Delta$, more precisely if $\mathcal{J} w^{2}>\Delta / 2$.

\section{Connection with the problem of a degenerate non-Kramers doublet}

Interestingly, this problem is dual to the problem of a nonKramers magnetic CEF ground doublet in a molecular field. In this case, we have $\left\langle 1\left|\mathbf{J}_{x, y, z}\right| 2\right\rangle \equiv 0,\left\langle 1\left|\mathbf{J}_{z}\right| 1\right\rangle=-\left\langle 2\left|\mathbf{J}_{z}\right| 2\right\rangle=\mu$, so that the matrix of the magnetic moment writes

$$
\mathbf{J}_{x}=0, \quad \mathbf{J}_{y}=0, \quad \mathbf{J}_{z}=\left(\begin{array}{cc}
-\mu & 0 \\
0 & \mu
\end{array}\right) .
$$

We shall also add fluctuations taking the form of the $y$ matrix element, induced for instance by a quadrupolar molecular field. For the sake of simplicity, we introduce one of the quadrupolar operators, written as

$$
\mathcal{O}=\left(\begin{array}{ll}
0 & w \\
w & 0
\end{array}\right)
$$

so that the Hamiltonian writes

$$
\mathcal{H}=\mathbf{h} . \mathbf{J}+q \mathcal{O}=\left(\begin{array}{cc}
-\Delta / 2 & y \\
y & \Delta / 2
\end{array}\right),
$$


which is identical to the two-singlet case, yet $\Delta$ now stands for $\Delta=2 \mu h$ and $y=q w$. Hence, the matrix of $\mathcal{O}$ writes

$$
\mathcal{O}=w\left(\begin{array}{cc}
\sin 2 \theta & \cos 2 \theta \\
\cos 2 \theta & -\sin 2 \theta
\end{array}\right),
$$

and the magnetic moment

$$
\mathbf{J}_{x}=0, \quad \mathbf{J}_{y}=0, \quad \mathbf{J}_{z}=\mu\left(\begin{array}{cc}
-\cos 2 \theta & \sin 2 \theta \\
\sin 2 \theta & \cos 2 \theta
\end{array}\right) .
$$

In other words, a soon as $\theta \neq 0$, the new states get a reduced magnetic moment and an emergent off-diagonal matrix element. This situation is the dual of the two-singlet problem:

$$
\begin{aligned}
\left|1^{\prime}\right\rangle & =\cos \theta|1\rangle+\sin \theta|2\rangle, \\
\left|2^{\prime}\right\rangle & =-\sin \theta|1\rangle+\cos \theta|2\rangle, \\
\left\langle 1^{\prime}\left|\mathbf{J}_{z}\right| 1^{\prime}\right\rangle & =-\mu \cos 2 \theta, \\
\left\langle 2^{\prime}\left|\mathbf{J}_{z}\right| 2^{\prime}\right\rangle & =\mu \cos 2 \theta, \\
\left\langle 1^{\prime}\left|\mathbf{J}_{z}\right| 2^{\prime}\right\rangle & =\mu \sin 2 \theta,
\end{aligned}
$$

with the following averaged magnetization and quadrupolar moment:

$$
\begin{aligned}
\mathbf{m}_{z} & =-\mu \frac{\Delta}{\sqrt{\Delta^{2}+4 y^{2}}} \tanh \frac{\sqrt{\Delta^{2}+4 y^{2}}}{2 T}, \\
o & =-w \frac{2 y}{\sqrt{\Delta^{2}+4 y^{2}}} \tanh \frac{\sqrt{\Delta^{2}+4 y^{2}}}{2 T} .
\end{aligned}
$$

The neutron cross section writes

$$
\begin{aligned}
I= & (\mu \cos 2 \theta)^{2} \delta(E) \\
& +\frac{1}{Z}(\mu \sin 2 \theta)^{2}\left[\delta(E-\epsilon)+e^{-\epsilon / T} \delta(E+\epsilon)\right]
\end{aligned}
$$

and thus also has inelastic peaks at $E= \pm \epsilon$ and a contribution at zero energy. At low temperature, we recover the same result as in the two-singlet model: the weight of the inelastic signal and of the elastic peak have opposite variations.

At the mean-field level, $h$ is replaced by a molecular field, $h=\mathcal{J} \mathbf{m}_{z}$, and $q=\mathcal{K} o$, so that the previous equations form a set of self-consistent equations. Depending on the relative strength of the two interactions $\mathcal{J}$ and $\mathcal{K}$, a magnetic or a nonmagnetic quadrupolar ground state may appear. In the low-temperature limit, one obtains

$$
\begin{aligned}
& \Delta=-2 \mu^{2} \mathcal{J} \frac{\Delta}{\sqrt{\Delta^{2}+4 y^{2}}} \tanh \frac{\sqrt{\Delta^{2}+4 y^{2}}}{2 T}, \\
& y=-2 w^{2} \mathcal{K} \frac{y}{\sqrt{\Delta^{2}+4 y^{2}}} \tanh \frac{\sqrt{\Delta^{2}+4 y^{2}}}{2 T} .
\end{aligned}
$$

\section{APPENDIX D: RPA CALCULATIONS}

\section{Hamiltonian, definitions, and conventions}

\section{a. Magnetic degrees of freedom}

In the following, we consider the generalized Heisenberg Hamiltonian:

$$
\begin{aligned}
\mathcal{H}= & \mathcal{H}_{\mathrm{CEF}}+\frac{1}{2} \sum_{i, j} \mathcal{J}_{i, j} \mathbf{J}_{i} \mathbf{J}_{j}+\sum_{i} g_{j} \mu_{B} \mathbf{J}_{i} \cdot h \\
& +\frac{1}{2} \sum_{i, j} \mathcal{K}_{i, j} \mathbf{O}_{i} \mathbf{O}_{j} .
\end{aligned}
$$

$H_{\mathrm{CEF}}$ is the crystal field Hamiltonian, $h$ is the magnetic field, $\mathcal{J}$ is a $(3 \times 3)$ interaction tensor which couples magnetic moments, and $\mathcal{K}_{i, j}$ is the interaction tensor between multipoles written as $O_{i}$ for the sake of simplicity. The site index $i$ is recast into an index $m$ denoting the unit cell and $i$ denoting the atomic site within the unit cell. Since the largest term in $\mathcal{H}$ is the crystal field Hamiltonian $\mathcal{H}_{\mathrm{CEF}}$, it is convenient to use the basis formed by the set of wave vectors $|a\rangle$,

$$
\mathcal{H}_{\mathrm{CEF}}|a\rangle=E_{a}|a\rangle,
$$

and the projection operators defined on this basis:

$$
|a\rangle\langle b|=X^{a b} .
$$

They are characterized by the commutation rules:

$$
\left[X_{i}^{a b}, X_{j}^{c d}\right]=\delta_{i, j}\left(X^{a d} \delta_{b c}-X^{c b} \delta_{d, a}\right) .
$$

Fourier-transformed operators are defined as

$$
\begin{gathered}
X_{k, i}^{a b}=\sum_{m} X_{m, i}^{a b} e^{i k R_{m}}, \\
{\left[X_{k, i}^{a b}, X_{k^{\prime}, j}^{c d}\right]=\delta_{k^{\prime},-k} \delta_{i, j}\left(X_{k, i}^{a d} \delta_{b c}-X_{k, i}^{c b} \delta_{d, a}\right) .} \\
\text { b. Lattice degrees of freedom }
\end{gathered}
$$

To take into account the interactions with lattice degrees of freedom (phonons), we consider the elastic Hamiltonian:

$$
\mathcal{H}_{\mathrm{el}}=\sum_{m, i} \frac{p_{m, i}^{2}}{2 M_{i}}+\frac{1}{2} \sum_{m, i, n, j} u_{m, i} \Gamma_{m, i, n, j} u_{n, j},
$$

where $\Gamma_{m, i, n, j}$ denote the force constants, $M_{i}$ the masses, and $u$ and $p$ the positions and momentum, respectively. In Fourier space, the dynamical matrix writes as

$$
\left(D_{k}\right)_{i, j}=\sum_{\Delta_{m, n}} \Gamma_{m, i, n, j}\left(\Delta_{m, n}\right) / \sqrt{M_{i} M_{j}} e^{i k \Delta_{m, n}} .
$$

$\Delta_{m, n}$ joins the unit cells labeled $m$ and $n$. We shall denote $\Omega_{k, s}^{2}$ the eigenvalues and $\mathbf{e}_{k, s}$ the eigenvectors of the dynamical matrix $D_{k}$. The index $s$ runs over the $3 L$ phonon modes of the unit cell containing $L$ atoms. The normal coordinate $\tilde{u}$ and its conjugated momentum $\tilde{p}$ are defined as

$$
\begin{aligned}
u_{k, i} & =\sum_{s} \mathbf{e}_{k, i, s} \sqrt{\frac{1}{M_{i}}} \tilde{u}_{k, s}, \\
p_{k, i} & =\sum_{s} \mathbf{e}_{k, i, s} \sqrt{M_{i}} \tilde{p}_{k, s} .
\end{aligned}
$$

Since the $\left\{\mathbf{e}_{k, s}\right\}$ form an orthogonal basis, one obtains

$$
\mathcal{H}_{\mathrm{el}}=\sum_{k, s} \frac{\tilde{p}_{k, s} \tilde{p}_{-k, s}}{2}+\frac{1}{2} \sum_{k, s} \tilde{u}_{k, s} \Omega_{k, s}^{2} \tilde{u}_{-k, s} .
$$

Using standard annihilation and creation operators,

$$
\begin{aligned}
& \tilde{u}_{k, s}=\sqrt{\frac{1}{2 \Omega_{k, s}}}\left(a_{-k, s}^{+}+a_{k, s}\right), \\
& \tilde{p}_{k, s}=i \sqrt{\frac{\Omega_{k, s}}{2}}\left(a_{k, s}^{+}-a_{-k, s}\right),
\end{aligned}
$$


with

$$
\left[\tilde{u}_{k, s}, \tilde{p}_{k, s}\right]=i,
$$

the elastic Hamiltonian eventually becomes

$$
\mathcal{H}_{\mathrm{el}}=\frac{1}{2} \sum_{k, s}\left(a_{k, s}^{+}+a_{-k, s}\right)\left(\begin{array}{cc}
\Omega_{k, s} & \\
& \Omega_{k, s}
\end{array}\right)\left(\begin{array}{c}
a_{k, s} \\
a_{-k, s}^{+}
\end{array}\right) .
$$

\section{c. Coupling}

The interaction between these degrees of freedom is modeled by a local coupling akin to a generalized Jahn-Teller effect:

$$
\mathcal{H}_{\mathrm{cpl}}=\sum_{m, i, t} v_{i, t=(a, b)} u_{m, i}\left(X_{m, i}^{a b}+X_{m, i}^{b a}\right) .
$$

It involves the displacement of the atom at site $i$ combined with an electronic transition from state $|a\rangle$ to state $|b\rangle$ at the same site; $v$ is a tensor (independent of $m$ ), whose microscopic origin remains to be described, and which carries the strength of this process. $t$ labels the transition from state $|b\rangle$ to $|a\rangle$, in Fourier space:

$$
\begin{aligned}
\mathcal{H}_{\mathrm{cpl}} & =\sum_{m, i, t, q, k} v_{i, t} e^{i(q+k) R_{m}} u_{q, i}\left(X_{k, i}^{a b}+X_{k, i}^{b a}\right) \\
& =\sum_{k, i, t} v_{i, t} u_{-k, i}\left(X_{k, i}^{a b}+X_{k, i}^{b a}\right) \\
& =\sum_{k, i, t} \tilde{v}_{k, i, s, t}\left(a_{-k, s}+a_{k, s}^{+}\right)\left(X_{k, i}^{a b}+X_{k, i}^{b a}\right) .
\end{aligned}
$$

$\tilde{v}$ is thus an effective tensor giving the strength of the coupling at site $i$ with the mode $s$ and transition $t$ :

$$
\tilde{v}_{k, i, s, t}=v_{i, t} \mathbf{e}_{k, i, s} \sqrt{\frac{1}{2 M_{i} \Omega_{k, s}}} .
$$

\section{Mean-field approximation}

Our approach is based on a mean-field approximation, performed on electronic and lattice degrees of freedom:

$$
\begin{aligned}
\mathcal{H} \approx & \mathcal{H}_{\mathrm{CEF}}+\sum_{m, i}\left(\sum_{n, j}\left\langle\mathbf{J}_{n, j}\right\rangle \mathbf{J}_{m, i, n, j}+g_{j} \mu_{B} h\right) \mathbf{J}_{m, i} \\
& +\left(\sum_{n, j}\left\langle\mathbf{O}_{n, j}\right\rangle K_{m, i, n, j}\right) \mathbf{O}_{m, i} \\
& +\sum_{m, i, t} v_{i, t=(a, b)}\left\langle u_{m, i}\right\rangle\left(X_{m, i}^{a b}+X_{m, i}^{b a}\right) \\
& +\sum_{m, i, t} v_{i, t=(a, b)} u_{m, i}\left\langle X_{m, i}^{a b}+X_{m, i}^{b a}\right\rangle .
\end{aligned}
$$

The average value $\left\langle u_{m, i}\right\rangle$ can be determined in a self-consistent way by minimizing the classical elastic energy $\mathcal{E}$ (taking into account the contribution from force constants only and not the inertia):

$$
\begin{aligned}
\mathcal{E}= & \sum_{m, i, t} v_{i, t=(a, b)} u_{m, i}\left|X_{m, i}^{a b}+X_{m, i}^{b a}\right\rangle \\
& +\frac{1}{2} \sum_{m, i, n, j} u_{m, i} \Gamma_{m, i, n, j} u_{n, j} .
\end{aligned}
$$

This mean-field step renormalizes the eigenvalues $E_{a}$ and eigenvectors $|a\rangle$, hence the projection operators. In terms of these new operators, the mean-field Hamiltonian writes

$$
\begin{aligned}
\mathcal{H} & =\sum_{k, i, a} E_{i, a} X_{k, i}^{a a}+\mathcal{V}+\mathcal{H}_{\mathrm{cpl}}+\mathcal{H}_{\mathrm{el}}, \\
\mathcal{V} & =\frac{1}{2} \sum_{m, i, n, j, a, b, c, d} \mathcal{V}_{m, i, n, j, a, b, c, d} X_{m, i}^{a b} X_{n, j}^{c d},
\end{aligned}
$$

with

$$
\begin{aligned}
\mathcal{V}_{m, i, n, j, a, b, c, d}= & \left\langle i, a\left|\mathbf{J}_{m, i}-\left\langle\mathbf{J}_{m, i}\right\rangle\right| i, b\right\rangle \mathcal{J}_{m, i, n, j}\langle j, c| \mathbf{J}_{n, j} \\
& -\left\langle\mathbf{J}_{n, j}\right\rangle|j, d\rangle+\left\langle i, a\left|\mathbf{O}_{m, i}-\left\langle\mathbf{O}_{m, i}\right\rangle\right| i, b\right\rangle \mathcal{K}_{m, i, n, j} \\
& \times\left\langle j, c\left|\mathbf{O}_{n, j}-\left\langle\mathbf{O}_{n, j}\right\rangle\right| j, d\right\rangle .
\end{aligned}
$$

\section{Green's function formalism}

To study this problem, we shall use the response function formalism. From a general point of view, the $R_{A B}$ response function is defined by

$$
R_{A B}=\langle\langle A, B\rangle\rangle=i \theta(t)\langle[A(t), B]\rangle .
$$

Deriving with respect to time, one obtains

$$
\begin{aligned}
\frac{d R_{A B}}{d t} & =i \delta(t)\langle[A, B]\rangle+i \theta(t)\left\langle\left[\frac{d A(t)}{d t}, B\right]\right\rangle \\
& =i \delta(t)\langle[A, B]\rangle+i \theta(t)\langle[-i[A, \mathcal{H}], B]\rangle \\
& =i \delta(t)\langle[A, B]\rangle-i R_{[A, \mathcal{H}] B} .
\end{aligned}
$$

Using the Fourier-transformed function,

$$
R_{A B}(\omega)=\lim _{\eta \rightarrow 0^{+}} \int d t e^{i \omega t-\eta t} \chi_{A B}(t),
$$

the equation of motion becomes

$$
(\omega+i \eta) R_{A B}=\langle[A, B]\rangle-R_{[A, \mathcal{H}], B} .
$$

Importantly, the magnetic susceptibility (and its imaginary part measured by neutron scattering) can be written in terms of these response functions as

$$
\chi_{i, j}=\sum_{t, t^{\prime}}\left(\left\langle i, a\left|\mathbf{J}_{\mathbf{i}}-\left\langle\mathbf{J}_{\mathbf{i}}\right\rangle\right| i, b\right\rangle\left\langle\mathbf{J}, a^{\prime}\left|\mathbf{J}_{\mathbf{j}}-\left\langle\mathbf{J}_{\mathbf{j}}\right\rangle\right| \mathbf{J}, b^{\prime}\right\rangle\right) R_{i, s \mid j, s^{\prime}}
$$

with

$$
R_{i, t=(a, b) \mid \mathbf{J}, t^{\prime}=\left(a^{\prime}, b^{\prime}\right)}=\left\langle\left\langle X_{k, i}^{a b}, X_{-k, j}^{a^{\prime} b^{\prime}}\right\rangle\right\rangle .
$$

With the matrix elements

$$
\mathbf{W}_{i, t}=\left\langle i, a\left|\mathbf{J}_{i}-\left\langle\mathbf{J}_{i}\right\rangle\right| i, b\right\rangle,
$$

one obtains

$$
\chi_{i, j}=\sum_{t, t^{\prime}} \mathbf{W}_{i, t} \mathbf{W}_{\mathbf{J}, t^{\prime}} R_{i, t \mid j, t^{\prime}},
$$


and in Fourier space,

$$
\chi(Q, \omega)=\sum_{i, j, t, t^{\prime}} e^{i Q \cdot\left(\vec{r}_{i}-\vec{r}_{j}\right)} \mathbf{W}_{i, t} \mathbf{W}_{\mathbf{J}, t^{\prime}} R_{i, t \mid j, t^{\prime}} .
$$

$Q$ is a reciprocal lattice vector (with respect to the mean-field unit cell). The $\vec{r}_{i}$ denote the equilibrium atomic positions in the same frame.

\section{Formal solution of the equations of motion}

a. No interactions: The bare susceptibility

Using the above formalism for $A=X_{k i}^{a b}$ and $B=X_{-k \mathbf{J}}^{a^{\prime} b^{\prime}}$, we get

$$
\begin{aligned}
{\left[X_{k i}^{a b}, E_{\mathbf{J}, \mu} X_{q \mathbf{J}}^{\mu \mu}\right] } & =E_{i, \mu}\left(X_{k, i}^{a \mu} \delta_{\mu, b}-X_{k, i}^{\mu b} \delta_{\mu, a}\right) \\
& =\left(E_{i, b}-E_{i, a}\right) X_{k i}^{a b} .
\end{aligned}
$$

Meanwhile

$$
\begin{aligned}
\left\langle\left[X_{k, i}^{a b}, X_{-k, j}^{a^{\prime} b^{\prime}}\right]\right\rangle & =\delta_{i, j}\left\langle\left(X_{k, i}^{a b^{\prime}} \delta_{b, a^{\prime}}-X_{k, i}^{a^{\prime} b} \delta_{b^{\prime}, a}\right)\right\rangle \\
& =\delta_{i, j}\left\langle\left(X_{k, i}^{a b^{\prime}} \delta_{a, b^{\prime}} \delta_{b, a^{\prime}}-X_{k, i}^{a^{\prime} b} \delta_{b^{\prime}, a} \delta_{b, a^{\prime}}\right)\right\rangle \\
& =\delta_{i, j} \delta_{a, b^{\prime}} \delta_{b, a^{\prime}}\left(p_{i, a}-p_{i, b}\right)
\end{aligned}
$$

with

$$
p_{i, a}=\exp \left(-E_{i, a} / T\right) / \sum_{b} \exp \left(-E_{i, b} / T\right) .
$$

With the convention

$$
\begin{aligned}
\gamma_{i, t} & =p_{i, a}-p_{i, b}, \\
\Delta_{i, t} & =E_{i, a}-E_{i, b}, \\
t & =b \rightarrow a, \\
\bar{t} & =a \rightarrow b,
\end{aligned}
$$

we have

$$
R_{k, i, t \mid-k, j, t^{\prime}}=\frac{\gamma_{i, t}}{\omega+i \eta-\Delta_{i, t}} \delta_{i, j} \delta_{t^{\prime}, \bar{t}}
$$

Defining the diagonal operators $\gamma, \Delta$, and $L$ with $L_{i, j, t, t^{\prime}}=$ $\delta_{i, j} \delta_{t^{\prime}, \bar{t}}$, we get

$$
R=\frac{\gamma}{\omega+i \eta-\Delta} L
$$

\section{b. The effect of magnetic interactions}

To take into account the effect of magnetic interactions, one can now calculate $\left[X_{k, i}^{\nu \mu}, \mathcal{V}\right]$ :

$$
\begin{aligned}
{\left[A, X_{q, \ell^{a}}^{a b} X_{-q, \ell^{\prime}}^{c d}\right]=} & {\left[X_{k, i}^{v \mu}, X_{q, \ell^{a b}}^{a b} X_{-q, \ell^{\prime}}^{c d}\right] } \\
= & {\left[\delta_{i, \ell} \delta_{q,-k}\left(X_{k, i}^{v b} \delta_{\mu, a}-X_{k, i}^{a \mu} \delta_{b, v}\right)+X_{q, \ell}^{a b} X_{k, i}^{v \mu}\right] X_{-q, \ell^{\prime}}^{c d}-X_{q, \ell}^{a b} X_{-q, \ell^{\prime}}^{c d} X_{k, i}^{v \mu} } \\
= & {\left[\delta_{i, \ell} \delta_{q,-k}\left(X_{k, i}^{v b} \delta_{\mu, a}-X_{k, i}^{a \mu} \delta_{b, v}\right) X_{-q, \ell^{\prime}}^{c d}+X_{q, \ell}^{a b} X_{k, i}^{v \mu} X_{-q, \ell^{\prime}}^{c d}\right]-X_{q, \ell}^{a b} X_{-q, \ell^{\prime}}^{c d} X_{k, i}^{v \mu} } \\
= & \delta_{i, \ell} \delta_{q,-k}\left(X_{k, i}^{v b} \delta_{\mu, a}-X_{k, i}^{a \mu} \delta_{b, \nu}\right) X_{-q, \ell^{\prime}}^{c d} \\
& +X_{q, \ell}^{a b}\left[\delta_{i, \ell^{\prime}} \delta_{q, k}\left(X_{k, i}^{v d} \delta_{\mu, c}-X_{k, i}^{c \mu} \delta_{d, v}\right)+X_{-q, \ell^{\prime}}^{c d} X_{k, i}^{v \mu}\right]-X_{q, \ell^{a}}^{a b} X_{-q, \ell^{\prime}}^{c d} X_{k, i}^{v \mu} \\
= & \delta_{i, \ell} \delta_{q,-k}\left(X_{k, i}^{v b} X_{-q, \ell^{\prime}}^{c d} \delta_{\mu, a}-X_{k, i}^{a \mu} X_{-q, \ell^{\prime}}^{c d} \delta_{b, v}\right) \\
& +\delta_{i, \ell^{\prime}} \delta_{q, k}\left(X_{q, \ell}^{a b} X_{k, i}^{v d} \delta_{\mu, c}-X_{q, \ell^{a}}^{a b} X_{k, i}^{c \mu} \delta_{d, v}\right),
\end{aligned}
$$

which is further approximated by

$$
\begin{aligned}
{\left[A, X_{q, \ell}^{a b} X_{-q, \ell^{\prime}}^{c d}\right]=} & \delta_{i, \ell} \delta_{q,-k}\left(\left\langle X_{k, i}^{v \nu}\right\rangle-\left\langle X_{k, i}^{\mu \mu}\right\rangle\right) \delta_{\nu, b} \delta_{\mu, a} X_{-q, \ell^{\prime}}^{c d} \\
& +\delta_{i, \ell^{\prime}} \delta_{q, k}\left(\left\langle X_{k, i}^{v \nu}\right\rangle-\left\langle X_{k, i}^{\mu \mu}\right\rangle\right) \delta_{\nu, d} \delta_{\mu, c} X_{q, \ell}^{a b},
\end{aligned}
$$

to write

$$
\begin{aligned}
{[A, \mathcal{V}] } & =\frac{\left(\left\langle X_{k, i}^{\nu \nu}\right\rangle-\left\langle X_{k, i}^{\mu \mu}\right\rangle\right)}{2}\left(\sum_{\ell^{\prime}, c, d} \mathcal{V}_{i, \ell^{\prime}, \mu, v, c, d} X_{k, \ell^{\prime}}^{c d}+\sum_{\ell, a, b} \mathcal{V}_{\ell, i, a, b, \mu, \nu} X_{k, \ell^{\prime}}^{a b}\right) \\
& =\left(\left\langle X_{k, i}^{\nu \nu}\right\rangle-\left\langle X_{k, i}^{\mu \mu}\right\rangle\right) \sum_{\ell^{\prime}, t^{\prime}} \mathcal{V}_{i, \ell^{\prime}, \bar{t}, t^{\prime}} X_{k, \ell^{\prime}}^{c d} .
\end{aligned}
$$

In a more compact form,

$$
\{(\omega-\Delta+i \eta) I+\gamma L \mathcal{V}\} R=\gamma L,
$$

where the operators $L$ and $\gamma$ are defined using the generalized $t$ index. Indeed, we notice that

$$
(L \mathcal{V})_{i, j, t, t^{\prime}}=\sum_{\ell, t^{\prime \prime}} L_{i, \ell, t, t^{\prime \prime}} \mathcal{V}_{\ell, j, t^{\prime \prime}, t^{\prime}}=\sum_{t^{\prime \prime}} \delta_{t^{\prime \prime}, \bar{t}} \mathcal{V}_{i, j, t^{\prime \prime}, t^{\prime}}=\mathcal{V}_{i, j, \bar{t}, t^{\prime}}
$$

\section{c. Lattice degrees of freedom}

The same reasoning applies to lattice degrees of freedom, using either $A=a_{k i}$ or $a_{-k i}^{+}, B=a_{k, j}^{+}$, and the standard boson commutation rules. Since

$$
\mathcal{H}_{\mathrm{el}}=\frac{1}{2} \sum_{k, s}\left(a_{k, s}^{+}+a_{-k, s}\right)\left(\begin{array}{cc}
\Omega_{k, s} & \\
& \Omega_{k, s}
\end{array}\right)\left(\begin{array}{c}
a_{k, s} \\
a_{-k, s}^{+}
\end{array}\right),
$$


we get

$$
\begin{aligned}
{\left[a_{k, s}, \mathcal{H}_{\mathrm{el}}\right] } & =\Omega_{k, s} a_{k, s}, \\
{\left[a_{-k, s}^{+}, \mathcal{H}_{\mathrm{el}}\right] } & =-\Omega_{k, s} a_{-k, s}^{+} .
\end{aligned}
$$

As a result, the full matrix of the response function writes

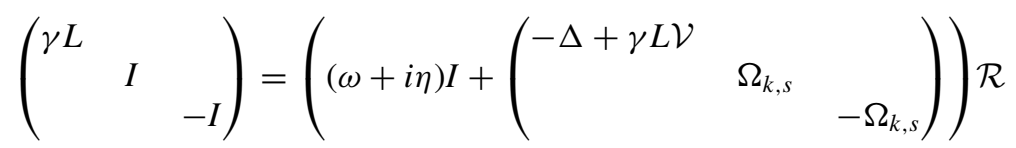

with

$$
\mathcal{R}=\left(\begin{array}{ccc}
\left\langle\left\langle X_{k, i}^{a b} X_{-k, j}^{c d}\right\rangle\right\rangle & \left\langle\left\langle X_{k, i}^{a b} a_{k, s^{\prime}}^{+}\right\rangle\right\rangle & \left\langle\left\langle X_{k, i}^{a b} a_{-k, s^{\prime}}\right\rangle\right\rangle \\
\left\langle\left\langle a_{k, s} X_{-k, j}^{c d}\right\rangle\right\rangle & \left\langle\left\langle a_{k, s} a_{k, s^{\prime}}^{+}\right\rangle\right\rangle & \left\langle\left\langle a_{k, s} a_{-k, s^{\prime}}\right\rangle\right\rangle \\
\left\langle\left\langle a_{-k, s}^{+} X_{-k, j}^{c d}\right\rangle\right\rangle & \left\langle\left\langle a_{-k, s}^{+} a_{k, s^{\prime}}^{+}\right\rangle\right. & \left\langle\left\langle a_{-k, s}^{+} a_{-k, s^{\prime}}\right\rangle\right\rangle
\end{array}\right) .
$$

These equations describe a resonant process where the phonon and the exciton hybridize to form new modes. The interaction is especially strong when the bare energies of the excitons and of the phonons are close to each other. At low temperature, coupling arises between the phonons and the first CEF transitions from the ground state. With increasing temperature, however, more and more transitions are thermally activated, hence increasing the possible coupling.
[1] C. Strohm, G. L. J. A. Rikken, and P. Wyder, Phys. Rev. Lett. 95, 155901 (2005).

[2] A. V. Inyushkin and A. N. TaIdenkov, JETP Lett. 86, 379 (2007).

[3] R. Berman, Thermal Conduction in Solids, Oxford Studies in Physics (Oxford University Press, 1976).

[4] H. Katsura, N. Nagaosa, and P. A. Lee, Phys. Rev. Lett. 104, 066403 (2010).

[5] M. Hirschberger, J. W. Krizan, R. J. Cava, and N. P. Ong, Science 348, 106 (2015).

[6] D. Watanabe, K. Sugii, M. Shimozawa, Y. Suzuki, T. Yajima, H. Ishikawa, Z. Hiroi, T. Shibauchi, Y. Matsuda, and M. Yamashita, Proc. Natl. Acad. Sci. USA 113, 8653 (2016).

[7] M. Hirschberger, R. Chisnell, Y. S. Lee, and N. P. Ong, Phys. Rev. Lett. 115, 106603 (2015).

[8] Y. Kasahara, K. Sugii, T. Ohnishi, M. Shimozawa, M. Yamashita, N. Kurita, H. Tanaka, J. Nasu, Y. Motome, T. Shibauchi, and Y. Matsuda, Phys. Rev. Lett. 120, 217205 (2018).

[9] T. Ideue, T. Kurumaji, S. Ishiwata, and Y. Tokura, Nat. Mater. 16, 797 (2017).

[10] L. Sheng, D. N. Sheng, and C. S. Ting, Phys. Rev. Lett. 96, 155901 (2006).

[11] Y. Kagan and L. A. Maksimov, Phys. Rev. Lett. 100, 145902 (2008).

[12] J.-S. Wang and L. Zhang, Phys. Rev. B 80, 012301 (2009).

[13] L. Zhang, J. Ren, J.-S. Wang, and B. Li, Phys. Rev. Lett. 105, 225901 (2010).

[14] T. Qin, J. Zhou, and J. Shi, Phys. Rev. B 86, 104305 (2012).

[15] M. Mori, A. Spencer-Smith, O. P. Sushkov, and S. Maekawa, Phys. Rev. Lett. 113, 265901 (2014).

[16] U. Loew, S. Zherlitsyn, K. Araki, M. Akatsu, Y. Nemoto, T. Goto, U. Zeitler, and B. Luethi, J. Phys. Soc. Jpn. 83, 044603 (2014).

[17] P. Thalmeier, Phys. Rev. B 80, 214421 (2009).
[18] A. Sytcheva, U. Low, S. Yasin, J. Wosnitza, S. Zherlitsyn, P. Thalmeier, T. Goto, P. Wyder, and B. Luthi, Phys. Rev. B 81, 214415 (2010).

[19] K. Araki, T. Goto, Y. Nemoto, T. Yanagisawa, and B. Luethi, Eur. Phys. J. B 61, 257 (2008).

[20] A. V. Inyushkin and A. N. Taldenkov, J. Exp. Theor. Phys. 111, 760 (2010).

[21] G. A. Slack and D. W. Oliver, Phys. Rev. B 4, 592 (1971).

[22] U. Walter, J. Phys. Chem. Solids 45, 401 (1984).

[23] J. Hammann, Acta Crystallogr., Sect. B 25, 1853 (1969).

[24] R. Wawrzynczak, B. Tomasello, P. Manuel, D. Khalyavin, M. D. Le, T. Guidi, A. Cervellino, T. Ziman, M. Boehm, G. J. Nilsen, and T. Fennell, Phys. Rev. B 100, 094442 (2019).

[25] R. Bidaux, A. Gavignet-Tillard, and J. Hammann, J. Phys. 34, 19 (1973).

[26] Y.-L. Wang and B. R. Cooper, Phys. Rev. 172, 539 (1968).

[27] S. Geller, Z. Kristallogr. 125, 1 (1967).

[28] J. Rodriguez-Carvajal, Physica B 192, 55 (1993).

[29] E. Kroumova, M. I. Aroyo, J. M. Perez-Mato, A. Kirov, C. Capillas, S. Ivantchev, and H. Wondratschek, Phase Transitions 76, 155 (2003).

[30] M. I. Aroyo, A. Kirov, C. Capillas, J. M. Perez-Mato, and H. Wondratschek, Acta Crystallogr., Sect. A 62, 115 (2006).

[31] S. Petit, L. Chaix, F. Damay, R. Galafassi, and J.-M. Zanotti, Vers l'utilisation du spectrometre temps de vol in6sharp en mode monocristaux, Institut Laue-Langevin (ILL), doi:10.5291/ILL-DATA.CRG-2616 (2019).

[32] M. Guillot, A. Marchand, V. Nekvasil, and F. Tcheou, J. Phys. C: Solid State Phys. 18, 3547 (1985).

[33] K. Kamazawa, D. Louca, R. Morinaga, T. J. Sato, Q. Huang, J. R. D. Copley, and Y. Qiu, Phys. Rev. B 78, 064412 (2008).

[34] H. W. Capel, Physica 31, 1152 (1965).

[35] M. Ball, M. J. M. Leask, D. Ryan, G. Garton, and W. P. Wolf, J. Appl. Phys. 32, S267 (1961).

[36] I. A. Kibalin, F. Damay, X. Fabreges, A. Gukasov, and S. Petit, Phys. Rev. Research 2, 033509 (2020). 
[37] J. Hammann and M. Ocio, Phys. Lett. A 55, 195 (1975).

[38] M. J. M. Leask, M. R. Wells, R. C. C. Ward, S. M. Hayden, and J. Jensen, J. Phys.: Condens. Matter 6, 505 (1994).

[39] J. Hammann and P. Manneville, J. Phys. 34, 615 (1973).

[40] A. Paddison et al., arXiv:1908.03530.

[41] A. J. Leggett, S. Chakravarty, A. T. Dorsey, M. P. A. Fisher, A. Garg, and W. Zwerger, Rev. Mod. Phys. 59, 1 (1987).

[42] U. Weiss, Quantum Dissipative Systems, 3rd ed., Series in Modern Condensed Matter Physics Vol. 13 (World Scientific Publishing Co. Ptc. Ltd., 2008).

[43] T. Fennell, M. Kenzelmann, B. Roessli, M. K. Haas, and R. J. Cava, Phys. Rev. Lett. 109, 017201 (2012).

[44] S. Petit, P. Bonville, J. Robert, C. Decorse, and I. Mirebeau, Phys. Rev. B 86, 174403 (2012).

[45] S. Petit, P. Bonville, I. Mirebeau, H. Mutka, and J. Robert, Phys. Rev. B 85, 054428 (2012).

[46] P. Bonville, I. Mirebeau, A. Gukasov, S. Petit, and J. Robert, Phys. Rev. B 84, 184409 (2011).
[47] S. Guitteny, J. Robert, P. Bonville, J. Ollivier, C. Decorse, P. Steffens, M. Boehm, H. Mutka, I. Mirebeau, and S. Petit, Phys. Rev. Lett. 111, 087201 (2013).

[48] T. Fennell, M. Kenzelmann, B. Roessli, H. Mutka, J. Ollivier, M. Ruminy, U. Stuhr, O. Zaharko, L. Bovo, A. Cervellino, M. K. Haas, and R. J. Cava, Phys. Rev. Lett. 112, 017203 (2014).

[49] M. Ruminy, S. Guitteny, J. Robert, L.-P. Regnault, M. Boehm, P. Steffens, H. Mutka, J. Ollivier, U. Stuhr, J. S. White, B. Roessli, L. Bovo, C. Decorse, M. K. Haas, R. J. Cava, I. Mirebeau, M. Kenzelmann, S. Petit, and T. Fennell, Phys. Rev. B 99, 224431 (2019).

[50] E. Constable, R. Ballou, J. Robert, C. Decorse, J. B. Brubach, P. Roy, E. Lhotel, L. Del-Rey, V. Simonet, S. Petit, and S. de Brion, Phys. Rev. B 95, 020415(R) (2017).

[51] H. R. Molavian, M. J. P. Gingras, and B. Canals, Phys. Rev. Lett. 98, 157204 (2007).

[52] H. Cao, A. Gukasov, I. Mirebeau, P. Bonville, C. Decorse, and G. Dhalenne, Phys. Rev. Lett. 103, 056402 (2009). 